\title{
Strauss's Radial Compactness and Nonlinear Elliptic Equation Involving a Variable Critical Exponent
}

\author{
Masato Hashizume ${ }^{1}$ and Megumi Sano $\mathbb{D}^{2}$ \\ ${ }^{1}$ Graduate School of Science and Engineering, Ehime University, 10-13 Dougohimata, Matsuyama-shi, Ehime-ken 790-8577, Japan \\ ${ }^{2}$ Department of Mathematics, Tokyo Institute of Technology, O-okayama, Meguro-ku, Tokyo 152-8551, Japan \\ Correspondence should be addressed to Megumi Sano; megumisano0609@gmail.com
}

Received 4 April 2018; Accepted 5 August 2018; Published 17 September 2018

Academic Editor: Dumitru Motreanu

Copyright (c) 2018 Masato Hashizume and Megumi Sano. This is an open access article distributed under the Creative Commons Attribution License, which permits unrestricted use, distribution, and reproduction in any medium, provided the original work is properly cited.

\begin{abstract}
We study existence of a nontrivial solution of $-\Delta_{p} u(x)+u(x)^{p-1}=u(x)^{q(x)-1}, u(x) \geq 0, x \in \mathbb{R}^{N}, u \in W_{\text {rad }}^{1, p}\left(\mathbb{R}^{N}\right)$, under some conditions on $q(x)$, especially, $\liminf _{|x| \rightarrow \infty} q(x)=p$. Concerning this problem, we firstly consider compactness and noncompactness for the embedding from $W_{\text {rad }}^{1, p}\left(\mathbb{R}^{N}\right)$ to $L^{q(x)}\left(\mathbb{R}^{N}\right)$. We point out that the decaying speed of $q(x)$ at infinity plays an essential role on the compactness. Secondly, by applying the compactness result, we show the existence of a nontrivial solution of the elliptic equation.
\end{abstract}

\section{Introduction and Main Results}

In this article, we consider the following nonlinear elliptic equation:

$$
\begin{aligned}
-\Delta_{p} u+u^{p-1} & =u^{q(\cdot)-1}, \quad u \geq 0 \text { in } \mathbb{R}^{N}, \\
u & \in W_{\mathrm{rad}}^{1, p}\left(\mathbb{R}^{N}\right)
\end{aligned}
$$

for $1<p<N$, where $\Delta_{p} u=\operatorname{div}\left(|\nabla u|^{p-2} \nabla u\right)$ is $p$-Laplacian and variable exponent $q(x)$ is a measurable function satisfying $q(x)>p, \liminf _{|x| \rightarrow \infty} q(x)=p$. $p(x)$-Laplacian type elliptic equation is one of the problems with variable exponent and this type equation on is studied by many researchers in both bounded domain case and unbounded domain case. In this paper, we refer the paper studying on $\mathbb{R}^{N}$, in several subjects: multiplicity of solutions (see, e.g., $[1,2]$ ), existence of solutions of equations involving several nonlinearities (see, e.g., [3, 4]), equations under periodic assumptions (see e.g. [5, 6]), and so on. Moreover, existence of solutions of (1) involving variable exponent

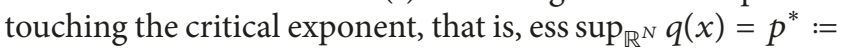
$N p /(N-p)$, is studied by $[7,8]$.

Concerning the classical Sobolev embedding in $\mathbb{R}^{N}$, it is well known that the embedding from $W_{\text {rad }}^{1, p}\left(\mathbb{R}^{N}\right)$ to $L^{p^{*}}\left(\mathbb{R}^{N}\right)$ is not compact. And the embedding from $W_{\text {rad }}^{1, p}\left(\mathbb{R}^{N}\right)$ to $L^{p}\left(\mathbb{R}^{N}\right)$ is not compact, either. In the viewpoint of the lack of compactness, consider the case where ess inf $\mathbb{R}_{\mathbb{R}^{N}} q(x)=p$ is natural as another critical case.

However, even for $p$-Laplace equation there are no results in this case. Thus we study problem (1) at the opening of this article. In this case, unlike the subcritical case, we need to overcome some difficulties to show the existence of a nontrivial solution of (1). We will explain them more precisely after Remark 5. Thus in advance of study of (1), we consider the related embedding to the equation. Namely, we study the embedding from $W_{\text {rad }}^{1, p}\left(\mathbb{R}^{N}\right)$ to $L^{q(x)}\left(\mathbb{R}^{N}\right)$.

We define the generalized Sobolev spaces $W^{k, p(x)}(\Omega)$ with variable exponents $p(x)$ according to [9]. For a domain $\Omega \subset$ $\mathbb{R}^{N}$ and a function $p \in L^{\infty}(\Omega)$ with $p(x) \geq 1$ we set

$$
\begin{aligned}
& L^{p(x)}(\Omega)=\{u \text { is a real measurable function on } \Omega \mid \\
& \left.\int_{\Omega}|u(x)|^{p(x)} d x<\infty\right\}, \\
& W^{k, p(x)}(\Omega)=\left\{u \in L^{p(x)}(\Omega)\left|D^{\alpha} u \in L^{p(x)}(\Omega),\right| \alpha \mid\right. \\
& \leq k\} .
\end{aligned}
$$


These $L^{p(x)}(\Omega)$ and $W^{k, p(x)}(\Omega)$ are Banach spaces with the following norms:

$$
\begin{aligned}
\|u\|_{p(x)} & =\inf \left\{\lambda>\left.0\left|\int_{\Omega}\right| \frac{u}{\lambda}\right|^{p(x)} d x \leq 1\right\}, \\
\|u\|_{W^{k, p(x)}} & =\|u\|_{p(x)}+\sum_{|\alpha| \leq k}\left\|D^{\alpha} u\right\|_{p(x)} .
\end{aligned}
$$

The Sobolev embedding theorem of $W^{k, p(x)}$ and related subjects have been well studied so far; see, e.g., [10-19], and we refer to the book [20]. For example, in bounded domain case, the Sobolev best constant with a variable critical exponent and the existence of extremals were studied in [21]; see also [22] in the Sobolev trace embedding case. One of the results in [18] is the existence of the compact embedding. They consider the situation when $p(x)$ is uniformly continuous on $\bar{\Omega}$ and $1<\operatorname{essinf}_{\bar{\Omega}} p(x) \leq \operatorname{esssup}_{\bar{\Omega}} p(x)<N / k$. Under this situation there exists a compact embedding from $W^{k, p(x)}(\Omega)$ to $L^{q(x)}(\Omega)$ for $q(x)$ satisfying $p(x) \leq q(x)$ a.e. in $\Omega$ and $\operatorname{essinf} \bar{\Omega}\left(p^{*}(x)-q(x)\right)>0$, where $p^{*}(x)=N p(x) /(N-$ $k p(x))$. On the other hand, for $W^{1, p}(\Omega)$ Kurata and Shioji [17] consider the critical case, that is, $\operatorname{ess}^{\sup _{\bar{\Omega}}} q(x)=p^{*}$. They showed that if there exist $x_{0} \in \Omega, C_{0}>0, \eta>0$ and $0<\ell<1$ such that ess $\sup _{\Omega \backslash B_{\eta}\left(x_{0}\right)} q(x)<p^{*}$ and

$$
q(x) \leq p^{*}-\frac{C_{0}}{|\log | x-\left.x_{0}\right|^{\ell}}
$$

for a.e. $x \in \Omega \cap B_{\eta}\left(x_{0}\right)$,

then the embedding from $W^{1, p}(\Omega)$ to $L^{q(x)}(\Omega)$ is compact. Conversely, if

$$
q(x) \geq p^{*}-\frac{C_{0}}{|\log | x-x_{0}||} \quad \text { for a.e. } x \in \Omega \cap B_{\eta}\left(x_{0}\right),
$$

then the embedding from $W^{1, p}(\Omega)$ to $L^{q(x)}(\Omega)$ is not compact.

When $\Omega=\mathbb{R}^{N}$, Strauss [23] and Lions [24] showed that the radial Sobolev space $W_{\mathrm{rad}}^{1, p}\left(\mathbb{R}^{N}\right)$ can be embedded to $L^{q}\left(\mathbb{R}^{N}\right)$ compactly for $q \in\left(p, p^{*}\right)$. In addition, related results are in $[25,26]$ and so on. In $p(x)$ case, under the same conditions as those of bounded domain case the compact embedding from $W_{\text {rad }}^{1, p(x)}\left(\mathbb{R}^{N}\right)$ to $L^{q(x)}\left(\mathbb{R}^{N}\right)$ is obtained for $q(x)$ satisfying ess inf $\mathbb{R}^{N}(q(x)-p(x))>0$ and

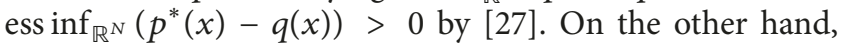
the critical case, that is, ess $\inf _{\mathbb{R}^{N}}(q(x)-p(x))=0$ or $\operatorname{ess} \inf _{\mathbb{R}^{N}}\left(p^{*}(x)-q(x)\right)=0$, has not been treated so far even if $p(x) \equiv p$. In this paper, we fix $p(x) \equiv p$. Our first study is to obtain a sufficiently condition of compactness and noncompactness of the embedding from $W_{\text {rad }}^{1, p}\left(\mathbb{R}^{N}\right)$ to $L^{q(x)}\left(\mathbb{R}^{N}\right)$ for variable exponent $q(x)$ which also includes the excluded case in [27]. Based on these results, as the second study we obtain a nontrivial solution of (1) under the compactness conditions with $\liminf _{|x| \rightarrow \infty} q(x)=p$.

Before introducing main results, we fix several notations. $B_{R}$ denote an open ball centered 0 with radius $R . \omega_{N-1}$ is an area of the unit sphere $\mathbb{S}^{N-1}$ in $\mathbb{R}^{N}$. Throughout this paper we assume that $q(x) \in L^{\infty}\left(\mathbb{R}^{N}\right)$ and $q(x) \geq 1$ for a.e. $x \in \mathbb{R}^{N}$. A letter $C$ denotes various positive constant. If $u$ is a radial function in $\mathbb{R}^{N}$, then we can write as $u(x)=\widetilde{u}(|x|)$ by some function $\widetilde{u}=\widetilde{u}(r)$ in $\mathbb{R}_{+}$. For simplicity we write $u(x)=u(|x|)$ with admitting some ambiguity.

Theorem 1 (noncompactness). If there exist positive constants $R$ and $C_{0}$ and an open set $\Gamma$ in $\mathbb{S}^{N-1}$ such that

$$
q(x) \leq p+\frac{C_{0}}{|\log | x||} \quad \text { for } x \in(R,+\infty) \times \Gamma ;
$$

then the embedding from $W_{\text {rad }}^{1, p}\left(\mathbb{R}^{N}\right)$ to $L^{q(x)}\left(\mathbb{R}^{N}\right)$ is not compact.

Theorem 2 (compactness). If there exist positive constants $r, R, C_{0}, C_{1}$, and $k, l \in(0,1)$ such that

$$
\begin{aligned}
& q(x) \leq p^{*}-\frac{C_{0}}{\left.|\log | x\right|^{k}} \quad \text { for } x \in B_{r}, \\
& q(x) \geq p+\frac{C_{1}}{|\log | x||^{\ell}} \quad \text { for } x \in \mathbb{R}^{N} \backslash B_{R},
\end{aligned}
$$

then the embedding from $W_{\text {rad }}^{1, p}\left(\mathbb{R}^{N}\right)$ to $L^{q(x)}\left(\mathbb{R}^{N}\right)$ is compact.

Remark 3. In Theorem 2, we do not need the constraint $p \leq$ $q(x) \leq p^{*} . W_{\text {rad }}^{1, p}\left(\mathbb{R}^{N}\right) \subset L^{q(x)}\left(\mathbb{R}^{N}\right)$ holds whenever $q(x)$ satisfies $q(x) \leq p^{*}$ in $B_{r}$ and $q(x) \geq p$ in $\mathbb{R}^{N} \backslash B_{R}$.

Theorem 4. Assume that $q(x)$ satisfies the hypotheses (7) and (8) in Theorem 2 and ess $\inf _{x \in B_{R}} q(x)>p$. Then there exists a nontrivial weak solution $u \in W_{\text {rad }}^{1, p}\left(\mathbb{R}^{N}\right)$ of (1) in the sense of

$$
\int_{\mathbb{R}^{N}}\left(|\nabla u|^{p-2} \nabla u \nabla \phi+u^{p-1} \phi-u^{q(x)-1} \phi\right) d x=0
$$

for any $\phi \in W_{\text {rad }}^{1, p}\left(\mathbb{R}^{N}\right)$.

Remark 5. If $q(x)$ is a radially symmetric function satisfying the hypotheses of Theorem 4, then we can show that the weak solution $u$ obtained in Theorem 4 satisfies $u \in C_{\text {loc }}^{1, \alpha}\left(\mathbb{R}^{N} \backslash\{0\}\right)$ and $u(x)>0$ for all $x \in \mathbb{R}^{N} \backslash\{0\}$. Indeed, since $u$ and $q(x)$ are radially symmetric, it follows that, for all $\phi \in W_{\text {rad }}^{1, p}\left(\mathbb{R}^{N}\right)$,

$$
\begin{aligned}
& \int_{0}^{\infty}\left(\left|u^{\prime}(r)\right|^{p-2} u^{\prime}(r) \phi^{\prime}(r)+u^{p-1} \phi-u^{q(r)-1} \phi\right) \\
& \cdot r^{N-1} d r=0,
\end{aligned}
$$

where $r=|x|$. If for any $\psi \in C_{c}^{\infty}\left(\mathbb{R}^{N}\right)$ we consider the radial function $\Psi(r)=\int_{\omega \in \mathbb{S}^{N-1}} \psi(r \omega) d S_{\omega}$, then we have

$$
\begin{aligned}
& \int_{\mathbb{R}^{N}}\left(|\nabla u|^{p-2} \nabla u \nabla \psi+u^{p-1} \psi-u^{q(x)-1} \psi\right) d x \\
& =\int_{0}^{\infty}\left(\left|u^{\prime}(r)\right|^{p-2} u^{\prime}(r) \Psi^{\prime}(r)+u^{p-1} \Psi-u^{q(r)-1} \Psi\right) \\
& \cdot r^{N-1} d r=0 .
\end{aligned}
$$


Therefore we see that $u$ satisfies (9) even for nonradial functions $\phi$. Finally, by Corollary of Theorem 2 in [28] we have $u \in C_{\text {loc }}^{1, \alpha}\left(\mathbb{R}^{N} \backslash\{0\}\right)$. And also, by Theorem 2.5.1 in [29] we have $u(x)>0$ for all $x \in \mathbb{R}^{N} \backslash\{0\}$.

We note the difficulties to obtain Theorem 4 caused by the condition ess $\inf _{x \in \mathbb{R}^{N}} q(x)=p$. Ambrosetti-Rabinowitz condition (AR) is well known in order to obtain a nontrivial weak solution to the following problem by mountain pass method:

$$
-\Delta_{p} u+|u|^{p-2} u=f(x, u) \quad \text { in } \mathbb{R}^{N} .
$$

There are $\mu>p$ and $M>0$

such that for $|u| \geq M$,

$$
0<\mu F(x, u) \leq u f(x, u),
$$

where $F(x, u):=\int_{0}^{u} f(x, s) d s$. Especially, condition (AR) has been used to establish not only the mountain pass structure of the energy functional but also the Palais-Smale condition. A weaker condition has also been considered, for instance, Liu-Wang [30] studied (SQ) which is called superquadratic condition.

$$
\lim _{|u| \rightarrow \infty} \frac{F(x, u)}{|u|^{p}}=\infty \quad \text { uniformly in } x \in \mathbb{R}^{N} .
$$

However, assuming that the nonlinear term $u(x)^{q(x)-1}$ in (1) is a special case of the general nonlinear term $f(x, u)$, this does not satisfy even condition (SQ) when ess $\inf _{x \in \mathbb{R}^{N}} q(x)=p$. From these facts, it seems to be difficult to confirm whether the energy functional $J$ (see Section 4) corresponding to (1) satisfies the Palais-Smale condition or not. In more detail, while the fact that bounded Palais-Smale sequence has a convergent subsequence is straightforward from Theorem 2 , boundedness of all Palais-Smale sequence is nontrivial. Besides that, satisfying the mountain pass structure for $J$ is not trivial since we cannot apply the fibering map method directly.

To overcome these difficulties, in Section 3, we construct a solution of (1) as a limit of mountain pass solutions of some elliptic equations approaching (1) in the sense of energy functional. In Section 4, we show another proof by using the variant of the mountain pass theorem. More precisely, by introducing the condition (C) (see Section 4) defined in [31] or [32] instead of the Palais-Smale condition, we obtain a solution of (1) in a different way from Section 3.

\section{Compactness and Noncompactness of the Embedding}

We prove Theorems 1 and 2. Before beginning the proof we recall the point-wise estimate and the compactness theorem introduced in $[23,24](p=2)$. For the reader's convenience, the proofs are in Appendix.
Proposition 6. For any $u \in W_{\text {rad }}^{1, p}\left(\mathbb{R}^{N}\right)$ we have

$$
\begin{aligned}
& |u(x)| \\
& \quad \leq\left(\frac{p}{\omega_{N-1}}\right)^{1 / p}|x|^{-(N-1) / p}\|u\|_{L^{p}\left(\mathbb{R}^{N}\right)}^{(p-1) / p}\|\nabla u\|_{L^{p}\left(\mathbb{R}^{N}\right)}^{1 / p} .
\end{aligned}
$$

Proposition 7. The embedding from $W_{\text {rad }}^{1, p}\left(\mathbb{R}^{N}\right)$ to $L^{q}\left(\mathbb{R}^{N}\right)$ is compact for $q \in\left(p, p^{*}\right)$.

Proof of Theorem 1. We shall show Theorem 1 in the same way as [17]. Set $r(x)=q(x)-p$ for $x \in \mathbb{R}^{N}$. Let $\phi \in C_{c}^{\infty}\left(\mathbb{R}^{N}\right)$ be a radial function satisfying $\phi \equiv 1$ on $B_{1 / 2}$ and $\operatorname{supp} \phi \subset B_{1}$. For $m \in \mathbb{N}$, we define $\phi_{m}(x)=m^{-N / p} \phi(x / m)$. Then for any $m \in \mathbb{N}$ we obtain

$$
\begin{aligned}
\left\|\phi_{m}\right\|_{L^{p}\left(\mathbb{R}^{N}\right)} & =\|\phi\|_{L^{p}\left(B_{1}\right)}, \\
\left\|\nabla \phi_{m}\right\|_{L^{p}\left(\mathbb{R}^{N}\right)} & =m^{-1}\|\nabla \phi\|_{L^{p}\left(B_{1}\right)} .
\end{aligned}
$$

Since $\left\{\phi_{m}\right\}_{m=1}^{\infty}$ is a bounded sequence in $W_{\mathrm{rad}}^{1, p}\left(\mathbb{R}^{N}\right)$ and $W_{\text {rad }}^{1, p}\left(\mathbb{R}^{N}\right)$ is reflexive (see, e.g., Proposition 3.20. in [33]), there exist a weakly convergent subsequence $\left\{\phi_{m_{j}}\right\}_{j=1}^{\infty}$ and $\phi_{\infty} \in W_{\text {rad }}^{1, p}\left(\mathbb{R}^{N}\right)$ such that $\phi_{m_{j}} \rightarrow \phi_{\infty}$ in $W_{\text {rad }}^{1, p}\left(\mathbb{R}^{N}\right)$ as $j \longrightarrow \infty$. By compactness of the embedding from $W_{\mathrm{rad}}^{1, p}\left(\mathbb{R}^{N}\right)$ to $L^{r}\left(\mathbb{R}^{N}\right)$ for $p<r<p^{*}$, we have $\phi_{m_{j}} \longrightarrow \phi_{\infty}$ in $L^{r}\left(\mathbb{R}^{N}\right)$ and $\phi_{m_{j}} \longrightarrow \phi_{\infty}$ a.e. in $\mathbb{R}^{N}$ which yields that $\phi_{\infty} \equiv 0$. On the other hand, we have

$$
\begin{aligned}
\int_{\mathbb{R}^{N}} & \left|\phi_{m}(x)\right|^{q(x)} d x \\
\quad & \int_{B_{m}} m^{-(N / p)(p+r(x))}\left|\phi\left(\frac{x}{m}\right)\right|^{q(x)} d x \\
& =\int_{B_{1}} m^{-(N / p) r(m y)}|\phi(y)|^{q(m y)} d y \\
& \geq \int_{B_{1 / 2} \backslash B_{1 / 4}} m^{-(N / p) r(m y)} d y .
\end{aligned}
$$

Since $\Gamma$ is open in $\mathbb{S}^{N-1}$, there exists a smooth subset $D \subset$ $\mathbb{S}^{N-1}$ such that $D \subset \Gamma$. By using the polar coordinates as $y=$ $s \omega\left(s>0, \omega \in \mathbb{S}^{N-1}\right)$ we obtain

$$
\begin{aligned}
& \int_{\mathbb{R}^{N}}\left|\phi_{m}(x)\right|^{q(x)} d x \\
& \geq \int_{1 / 4}^{1 / 2} \int_{\omega \in D} m^{-(N / p) r(m s \omega)} s^{N-1} d s d S_{\omega} .
\end{aligned}
$$

By the assumption (6), we obtain $r(m s \omega) \leq C_{0}|\log m s|^{-1}$ for large $m, s \in(1 / 4,1 / 2)$, and $\omega \in D \subset \Gamma$. Moreover for $s \epsilon$ $(1 / 4,1 / 2)$ and large $m$, it holds that $\log m s=\log m+\log s \geq$ 
$(1 / 2) \log m$ which yields

$$
r(m s \omega) \leq \frac{2 C_{0}}{\log m}
$$

Therefore we obtain

$$
\begin{aligned}
\int_{\mathbb{R}^{N}}\left|\phi_{m}(x)\right|^{q(x)} d x \\
\geq \int_{1 / 4}^{1 / 2} \int_{\omega \in D} e^{-(N / p) \log m\left(2 C_{0} / \log m\right)} s^{N-1} d s d S_{\omega} \\
=\mathscr{H}^{N-1}(D) e^{-2 C_{0} N / p} \frac{2^{-N}-4^{-N}}{N}>0
\end{aligned}
$$

for large $m$, where $\mathscr{H}^{d}$ is the $d$-dimensional Hausdorff measure. Thus, if we assume that the embedding from $W_{\text {rad }}^{1, p}\left(\mathbb{R}^{N}\right)$ to $L^{q(x)}\left(\mathbb{R}^{N}\right)$ is compact, then we have $\int_{\mathbb{R}^{N}}\left|\phi_{\infty}\right|^{q(x)} d x>0$ which contradicts $\phi_{\infty} \equiv 0$. Hence the embedding from $W_{\text {rad }}^{1, p}\left(\mathbb{R}^{N}\right)$ to $L^{q(x)}\left(\mathbb{R}^{N}\right)$ is not compact.

Proof of Theorem 2 . We assume that $r<R$ without loss of generality. Let $\left\{u_{m}\right\}_{m=1}^{\infty}$ be a bounded sequence in $W_{\text {rad }}^{1, p}\left(\mathbb{R}^{N}\right)$. We shall show the existence of a strongly convergence subsequence of $\left\{u_{m}\right\}_{m=1}^{\infty}$ in $L^{q(x)}\left(\mathbb{R}^{N}\right)$. By the reflexivity of $W_{\text {rad }}^{1, p}\left(\mathbb{R}^{N}\right)$, there exist a subsequence $\left\{u_{m_{j}}\right\}_{j=1}^{\infty}$ and $u_{0} \in$ $W_{\text {rad }}^{1, p}\left(\mathbb{R}^{N}\right)$ such that $u_{m_{j}} \rightarrow u_{0}$ in $W_{\text {rad }}^{1, p}\left(\mathbb{R}^{N}\right)$ as $j \longrightarrow \infty$. Especially it also holds that $u_{m_{j}} \rightarrow u_{0}$ in $W^{1, p}\left(\mathbb{R}^{N}\right)$ as $j \longrightarrow$ $\infty$. And also, by Proposition 7 we have $u_{m_{j}} \longrightarrow u_{0}$ in $L^{q}\left(\mathbb{R}^{N}\right)$ for any $q \in\left(p, p^{*}\right)$ and

$$
u_{m_{j}} \longrightarrow u_{0} \quad \text { a.e. in } \mathbb{R}^{N} \text { as } j \longrightarrow \infty \text {. }
$$

Furthermore, $\left\{\left.u_{m_{j}}\right|_{B_{r}}\right\}_{j=1}^{\infty} \subset W^{1, p}\left(B_{r}\right)$ is a bounded sequence and the embedding from $W^{1, p}\left(B_{r}\right)$ to $L^{q(x)}\left(B_{r}\right)$ is compact by the assumption (7) (see Remark 2 in [17]). Thus there exist a subsequence of $\left\{\left.u_{m_{j}}\right|_{B_{r}}\right\}_{j=1}^{\infty}$ (we use $\left\{\left.u_{m_{j}}\right|_{B_{r}}\right\}_{j=1}^{\infty}$ again for simplicity) and $v_{0} \in L^{q(x)}\left(B_{r}\right)$ such that the following hold true:

$$
\begin{aligned}
& \left.u_{m_{j}}\right|_{B_{r}} \rightarrow v_{0} \quad \text { in } W^{1, p}\left(B_{r}\right), \\
& \left.u_{m_{j}}\right|_{B_{r}} \longrightarrow v_{0} \text { in } L^{q(x)}\left(B_{r}\right) \text {, } \\
& \left.u_{m_{j}}\right|_{B_{r}} \longrightarrow v_{0} \text { in } L^{p}\left(B_{r}\right), \\
& \left.u_{m_{j}}\right|_{B_{r}} \longrightarrow v_{0} \quad \text { a.e. in } B_{r} \\
& \text { as } j \longrightarrow \infty \text {. }
\end{aligned}
$$

By (19) and (20), we can check that $\left.u_{0}\right|_{B_{r}}=v_{0}$ a.e. in $B_{r}$ which yields

$$
\left.\left.u_{m_{j}}\right|_{B_{r}} \longrightarrow u_{0}\right|_{B_{r}} \text { in } L^{q(x)}\left(B_{r}\right) \text { as } j \longrightarrow \infty \text {. }
$$

In the similar way as above, we also obtain the following:

$$
\begin{aligned}
& \left.\left.u_{m_{j}}\right|_{B_{K} \backslash B_{r}} \rightarrow u_{0}\right|_{B_{K} \backslash B_{r}} \text { in } W_{\mathrm{rad}}^{1, p}\left(B_{K} \backslash B_{r}\right), \\
& \left.\left.u_{m_{j}}\right|_{B_{K} \backslash B_{r}} \longrightarrow u_{0}\right|_{B_{K} \backslash B_{r}} \text { in } L^{s}\left(B_{K} \backslash B_{r}\right) \text {, } \\
& \left.\left.u_{m_{j}}\right|_{B_{K} \backslash B_{r}} \longrightarrow u_{0}\right|_{B_{K} \backslash B_{r}} \text { a.e. in } B_{K} \backslash B_{r}
\end{aligned}
$$

for any $K>0$ and any $s \geq 1$ as $j \longrightarrow \infty$ since the embedding from $W_{\mathrm{rad}}^{1, p}\left(B_{K} \backslash B_{r}\right)$ to $L^{s}\left(B_{K} \backslash B_{r}\right)$ is compact for any $K$, $s$.

Set $v_{m_{j}}:=u_{m_{j}}-u_{0}$. In order to make good use of (22) and (23) we divide $\int_{\mathbb{R}^{N}}\left|v_{m_{j}}(x)\right|^{q(x)} d x$ into three terms as follows:

$$
\begin{aligned}
\int_{\mathbb{R}^{N}}\left|v_{m_{j}}(x)\right|^{q(x)} d x= & \int_{B_{r}}\left|v_{m_{j}}(x)\right|^{q(x)} d x \\
& +\int_{B_{K} \backslash B_{r}}\left|v_{m_{j}}(x)\right|^{q(x)} d x \\
& +\int_{\mathbb{R}^{N} \backslash B_{K}}\left|v_{m_{j}}(x)\right|^{q(x)} d x \\
= & I_{1}(j)+I_{2}(j, K)+I_{3}(j, K),
\end{aligned}
$$

where $K$ is sufficiently large.

Firstly, by (22) we have

$$
I_{1}(j)=o(1) \quad \text { as } j \longrightarrow \infty \text {. }
$$

Next, for $I_{2}(j, K)$ we have

$$
\begin{aligned}
I_{2}(j, K)= & \int_{B_{K} \backslash B_{r}}\left|v_{m_{j}}(x)\right|^{q(x)} d x \\
\leq & \int_{B_{K} \backslash B_{r}}\left|v_{m_{j}}(x)\right| d x \\
& +\int_{B_{K} \backslash B_{r}}\left|v_{m_{j}}(x)\right|^{\left.\|q\|_{L^{\infty}} \mathbb{R}^{N}\right)} d x .
\end{aligned}
$$

Thus, by (23) we obtain

$$
I_{2}(j, K)=o(1) \quad \text { as } j \longrightarrow \infty \text { for fixed } K>0 .
$$

Finally we shall estimate $I_{3}(j, K)$. Since

$$
\begin{aligned}
\left|v_{m_{j}}(x)\right| & \leq\left(\frac{p}{\omega_{N-1}}\right)^{1 / p}\left\|v_{m_{j}}\right\|_{W^{1, p\left(\mathbb{R}^{N}\right)}}|x|^{-(N-1) / p} \\
& \leq C|x|^{-(N-1) / p}
\end{aligned}
$$


by Proposition 6 and the boundedness of $\left\{v_{m_{j}}\right\}_{j=1}^{\infty}$, we can assume $\left|v_{m_{j}}(x)\right| \leq 1$ for $x \in \mathbb{R}^{N} \backslash B_{K}$ with large $K$. Therefore by assumption (8) we obtain

$$
\begin{aligned}
& I_{3}(j, K)=\int_{\mathbb{R}^{N} \backslash B_{K}}\left|v_{m_{j}}\right|^{q(x)} d x \\
& \leq \int_{\mathbb{R}^{N} \backslash B_{K}}\left|v_{m_{j}}\right|^{p+C_{1}(\log |x|)^{-\ell}} d x \\
& \leq \sum_{n=2}^{\infty} \int_{B_{K^{n}} \backslash B_{K^{n-1}}}\left|v_{m_{j}}\right|^{p+C_{1}(n \log K)^{-\ell}} d x \\
& \leq \sum_{n=2}^{\infty} \int_{B_{K^{n}} \backslash B_{K^{n-1}}}\left|v_{m_{j}}\right|^{p}\left(C|x|^{-(N-1) / p}\right)^{C_{1}(n \log K)^{-\ell}} d x \\
& \leq C^{C_{1}(2 \log K)^{-\ell}}\left\|v_{m_{j}}\right\|_{W^{1, p}\left(\mathbb{R}^{N}\right)}^{p} \\
& \cdot \sum_{n=2}^{\infty} K^{-((N-1) / p)(n-1) C_{1}(n \log K)^{-\ell}} \leq C \sum_{n=2}^{\infty} \delta_{1}^{(n-1)^{1-\ell}} \\
& =C \sum_{n=1}^{\infty} \delta_{1}^{n^{1-\ell}}
\end{aligned}
$$

where $\delta_{1}=\delta_{1}(K):=K^{-((N-1) / p) C_{1}(\log K)^{-\ell}} \longrightarrow 0$ as $K \longrightarrow \infty$. Since $\sum_{n=1}^{\infty} \delta_{1}^{n^{1-\ell}}=\delta_{1}+\int_{1}^{\infty} \delta_{1}^{x^{1-\ell}} d x<\infty$ for each $\delta_{1} \in(0,1)$, we have

$$
\sum_{n=1}^{\infty} \delta_{1}^{n^{1-\ell}} \longrightarrow 0 \quad \text { as } K \longrightarrow \infty
$$

Hence we have

$$
I_{3}(j, K)=o(1) \quad \text { uniformly in } j \text { as } K \longrightarrow \infty .
$$

We go back to (24) and by (25), (27), and (31) we have

$$
\lim _{j \rightarrow \infty} \int_{\mathbb{R}^{N}}\left|v_{m_{j}}(x)\right|^{q(x)} d x=0
$$

As a consequence we obtain $u_{m_{j}} \longrightarrow u_{0}$ in $L^{q(x)}\left(\mathbb{R}^{N}\right)$.

\section{Approximation Method: Proof of Theorem 4}

In this section, we show Theorem 4 by using Theorem 2 . First, we prepare the mountain pass theorem (Theorem 8) introduced in $[34,35]$ and so on which are based on [36]. Let $V$ be a Banach space and $E \in C^{1}(V, \mathbb{R})$. We define a PalaisSmale sequence for $E$ as $\left\{u_{m}\right\} \subset V$ satisfying $\left|E\left(u_{m}\right)\right| \leq c$ uniformly in $m$, and $E^{\prime}\left(u_{m}\right) \longrightarrow 0$ in $V^{*}$, where $E^{\prime}(\cdot)$ is Fréchet derivative and $V^{*}$ is the dual space of $V$. We say that $E$ satisfies (P.-S.) condition if any Palais-Smale sequence has a strongly convergent subsequence.

Theorem 8 (see $[34,35])$. Suppose $E \in C^{1}(V, \mathbb{R})$ satisfies (P.S.) condition. Assume that

(i) $E(0)=0$ (ii) There exist $\rho>0, \alpha>0$ such that $E(u) \geq \alpha$ for any $u \in V$ with $\|u\|=\rho$.

(iii) There exists $u_{1} \in V$ such that $\left\|u_{1}\right\| \geq \rho$ and $E\left(u_{1}\right)<\alpha$. Define

$$
P=\left\{p \in C([0,1], V) \mid p(0)=0, p(1)=u_{1}\right\} .
$$

Then

$$
\beta=\inf _{p \in P} \sup _{0 \leq t \leq 1} E(p(t))
$$

is a critical value.

Proof of Theorem 4 .

Step 1. We may assume that $R$ in the hypotheses of Theorem 2 is sufficiently large such that ess inf $x_{x \in B_{R}} q(x) \geq p+$ $C_{1}(\log R)^{-\ell}$. Because, if not, we take a sufficiently large $\widetilde{R}$ which satisfies it instead of $R$. For $m \in \mathbb{N}$ let $\left\{R_{m}\right\}$ be a sequence such that $R_{1}=R$ and $R_{m} \longrightarrow \infty$ as $m \longrightarrow \infty$. Then we set functions as

$$
\begin{aligned}
& q_{m}(x) \\
& = \begin{cases}q(x) & \text { if } q(x) \geq p+C_{1}\left(\log R_{m}\right)^{-\ell}, \\
p+C_{1}\left(\log R_{m}\right)^{-\ell} & \text { if } q(x)<p+C_{1}\left(\log R_{m}\right)^{-\ell} .\end{cases}
\end{aligned}
$$

Define a functional $J_{m}$ from $W_{\text {rad }}^{1, p}\left(\mathbb{R}^{N}\right)$ to $\mathbb{R}$ by

$$
\begin{aligned}
J_{m}(u)= & \frac{1}{p} \int_{\mathbb{R}^{N}}\left(|\nabla u|^{p}+|u|^{p}\right) d x \\
& -\int_{\mathbb{R}^{N}} \frac{1}{q_{m}(x)} u_{+}^{q_{m}(x)} d x .
\end{aligned}
$$

We can check that $J_{m} \in C^{1}\left(W_{\text {rad }}^{1, p}\left(\mathbb{R}^{N}\right), \mathbb{R}\right)$. Indeed, for fixed $m$ and any $u, \phi \in W_{\text {rad }}^{1, p}\left(\mathbb{R}^{N}\right)$

$$
\begin{aligned}
D_{G} J_{m}(u)[\phi]:= & \left.\frac{d}{d t}\right|_{t=0} J_{m}(u+t \phi) \\
= & \int_{\mathbb{R}^{N}}|\nabla u|^{p-2} \nabla u \cdot \nabla \phi+|u|^{p-2} u \phi d x \\
& -\int_{\mathbb{R}^{N}} u_{+}^{q_{m}(x)-1} \phi d x .
\end{aligned}
$$

Then we see that $J_{m}$ is Gâteaux differentiable in $W_{\mathrm{rad}}^{1, p}\left(\mathbb{R}^{N}\right)$. By the Vitali convergence theorem, we see that $D_{G} J_{m}$ is continuous from $W_{\text {rad }}^{1, p}\left(\mathbb{R}^{N}\right)$ to its dual space $\left(W_{\text {rad }}^{1, p}\left(\mathbb{R}^{N}\right)\right)^{*}$. Hence $J_{m} \in C^{1}\left(W_{\mathrm{rad}}^{1, p}\left(\mathbb{R}^{N}\right), \mathbb{R}\right)$. Moreover, for each $m, J_{m}$ satisfies as follows:

(i) $J_{m}$ satisfies (P.-S.) condition.

(ii) $J_{m}(0)=0$,

(iii) There exist positive constants $\alpha, \rho$ such that $J_{m}(u) \geq \alpha$ for any $u \in W_{\text {rad }}^{1, p}\left(\mathbb{R}^{N}\right)$ with $\|u\|_{W^{1, p}\left(\mathbb{R}^{N}\right)}=\rho$, 
(iv) There exists $v \in W_{\text {rad }}^{1, p}\left(\mathbb{R}^{N}\right)$ such that $\|v\|_{W^{1, p}\left(\mathbb{R}^{N}\right)} \geq$ $\rho, J_{m}(v)<\alpha$.

By Theorem 8 there exists a critical point $u_{m} \in W_{\text {rad }}^{1, p}\left(\mathbb{R}^{N}\right)$ of $J_{m}$ such that

$$
J_{m}\left(u_{m}\right)=\beta_{m},
$$

where $\beta_{m}$ is defined in the same way as $\beta$ in Theorem 8 . Thus $u_{m}$ is a nontrivial weak solution of

$$
-\Delta_{p} w+|w|^{p-2} w=w_{+}^{q_{m}(x)-1} \quad \text { in } \mathbb{R}^{N} .
$$

We can also see that $u_{m} \geq 0$ by multiplying both sides of (39) by $\left(u_{m}\right)_{-}$.

Proposition 9. $\left\{u_{m}\right\}$ is bounded in $W_{\text {rad }}^{1, p}\left(\mathbb{R}^{N}\right)$.

We will prove this proposition at the end of this section.

Step 2. Since $\left\{u_{m}\right\}$ is a bounded sequence, there exists $u_{0} \in$ $W_{\text {rad }}^{1, p}\left(\mathbb{R}^{N}\right)$ such that $u_{m} \rightarrow u_{0}$ weakly in $W_{\text {rad }}^{1, p}\left(\mathbb{R}^{N}\right)$. Put

$$
\begin{aligned}
G_{m} & \\
= & \left\langle\left|\nabla u_{m}\right|^{p-2} \nabla u_{m}-\left|\nabla u_{0}\right|^{p-2} \nabla u_{0}, \nabla u_{m}-\nabla u_{0}\right\rangle_{\mathbb{R}^{N}} \\
& +\left(u_{m}^{p-1}-u_{0}^{p-1}\right)\left(u_{m}-u_{0}\right) .
\end{aligned}
$$

Then we have

$$
\begin{aligned}
\int_{\mathbb{R}^{N}} & G_{m} d x \\
= & \int_{\mathbb{R}^{N}}\left(\left|\nabla u_{m}\right|^{p}+u_{m}^{p}\right) d x \\
& \quad-\int_{\mathbb{R}^{N}}\left(\left|\nabla u_{m}\right|^{p-2} \nabla u_{m} \nabla u_{0}+u_{m}^{p-1} u_{0}\right) d x+h_{m},
\end{aligned}
$$

where $h_{m}=\int_{\mathbb{R}^{N}}\left[\left|\nabla u_{0}\right|^{p-2} \nabla u_{0}\left(\nabla u_{0}-\nabla u_{m}\right)+u_{0}^{p-1}\left(u_{0}-\right.\right.$ $\left.\left.u_{m}\right)\right] d x=o(1)$ as $m \longrightarrow \infty$. Moreover, from (56) and (57) in the proof of Proposition 9 it follows that

$$
\begin{aligned}
\int_{\mathbb{R}^{N}} & \left(\left|\nabla u_{m}\right|^{p}+u_{m}^{p}\right) d x \\
- & \int_{\mathbb{R}^{N}}\left(\left|\nabla u_{m}\right|^{p-2} \nabla u_{m} \nabla u_{0}+u_{m}^{p-1} u_{0}\right) d x \\
= & \int_{\mathbb{R}^{N}}\left(u_{m}\right)_{+}^{q_{m}(x)-1}\left(\left(u_{m}\right)_{+}-u_{0}\right) d x \\
\leq & 2\left\|u_{m}^{q_{m}}(x)-1\right\|_{q(x) /(q(x)-1)}\left\|u_{m}-u_{0}\right\|_{q(x)} \\
= & 2\left\|u_{m}\right\|_{q(x)}\left\|u_{m}-u_{0}\right\|_{q(x)}
\end{aligned}
$$

by the generalized Hölder inequality (see, e.g., [9] Theorem 2.1). By the boundedness of $\left\{u_{m}\right\}$ in $W_{\text {rad }}^{1, p}\left(\mathbb{R}^{N}\right)$ and Theorem 2 we have $\left\|u_{m}\right\|_{q(x)}\left\|u_{m}-u_{0}\right\|_{q(x)}=o(1)$ as $m \longrightarrow \infty$. Hence

$$
\int_{\mathbb{R}^{N}} G_{m} d x=o(1)
$$

as $m \longrightarrow \infty$. Recall that, for $p \geq 1, a, b \in \mathbb{R}^{d}$, we have

$$
\begin{aligned}
& \left\langle|b|^{p-2} b-|a|^{p-2} a, b-a\right\rangle \\
& \geq \begin{cases}2^{2-p}|b-a|^{p} & \text { if } p \geq 2, \\
(p-1)|b-a|^{2}\left(1+|a|^{2}+|b|^{2}\right)^{(p-2) / 2} & \text { if } 1 \leq p \leq 2 .\end{cases}
\end{aligned}
$$

From this inequality and (43) it follows that

$$
\int_{\mathbb{R}^{N}}\left(\left|\nabla u_{m}-\nabla u_{0}\right|^{p}+\left|u_{m}-u_{0}\right|^{p}\right) d x=o(1)
$$

which is equivalent to $u_{m} \longrightarrow u_{0}$ strongly in $W^{1, p}\left(\mathbb{R}^{N}\right)$. Thus $u_{0}$ satisfies

$$
-\Delta_{p} u_{0}+u_{0}^{p-1}=u_{0}^{q(x)-1}, \quad u_{0} \geq 0 \text { in } \mathbb{R}^{N}
$$

Step 3. Finally, we have to show $u_{0} \not \equiv 0$. From the boundedness of $\left\{u_{m}\right\}$ and Proposition 6 , we see that $u_{m} \leq 1$ in $\mathbb{R}^{N} \backslash B_{L}$ for large $L$. Therefore we have

$$
\begin{aligned}
& \int_{\mathbb{R}^{N}}\left(\left|\nabla u_{m}\right|^{p}+u_{m}^{p}\right) d x=\int_{\mathbb{R}^{N}} u_{m}^{q_{m}(x)} d x \\
& \leq \int_{\mathbb{R}^{N}} u_{m}^{p} d x+\int_{B_{r}} u_{m}^{p^{*}} d x+\int_{B_{L} \backslash B_{r}} u_{m}^{\|q\|_{L^{\infty}}\left(\mathbb{R}^{N}\right)} d x .
\end{aligned}
$$

By the Sobolev inequality it follows that

$$
\begin{aligned}
\int_{B_{r}} u_{m}^{p^{*}} d x & \leq \int_{\mathbb{R}^{N}} u_{m}^{p^{*}} d x \\
& \leq S^{-p^{*} / p}\left(\int_{\mathbb{R}^{N}}\left|\nabla u_{m}\right|^{p} d x\right)^{p^{*} / p} .
\end{aligned}
$$

Moreover, we have

$$
\begin{aligned}
& \int_{B_{L} \backslash B_{r}} u_{m}^{\|q\|_{L^{\infty}}\left(\mathbb{R}^{N}\right)} d x \\
& \leq C\left[\int_{B_{L} \backslash B_{r}}\left(\left|\nabla u_{m}\right|^{p}+\left|u_{m}\right|^{p}\right) d x\right]^{\|q\|_{L^{\infty}\left(\mathbb{R}^{N}\right)} / p} \\
& \leq C\left[\int_{B_{L} \backslash B_{r}}\left|\nabla u_{m}\right|^{p} d x+\left(\int_{B_{L} \backslash B_{r}}\left|u_{m}\right|^{p^{*}} d x\right)^{p / p^{*}}\right. \\
& \left.\cdot\left|B_{L} \backslash B_{r}\right|^{1-p / p^{*}}\right]^{\|q\|_{L^{\infty}\left(\mathbb{R}^{N}\right)} / p} \\
& \leq C\left(\int_{\mathbb{R}^{N}}\left|\nabla u_{m}\right|^{p} d x\right)^{\|q\|_{L^{\infty\left(\mathbb{R}^{N}\right)} / p} .}
\end{aligned}
$$

Put $q_{*}:=\min \left\{p^{*},\|q\|_{L^{\infty}\left(\mathbb{R}^{N}\right)}\right\}$. From (47), (48), and (49), we obtain

$$
C \leq\left(\int_{\mathbb{R}^{N}}\left|\nabla u_{m}\right|^{p} d x\right)^{\left(q_{*}-p\right) / p},
$$


where we used that $u_{m} \not \equiv 0$. By Theorem 2 we have

$$
\begin{aligned}
C & \leq \lim _{m \rightarrow \infty} \int_{\mathbb{R}^{N}}\left|\nabla u_{m}\right|^{p} d x \\
& =\lim _{m \rightarrow \infty} \int_{\mathbb{R}^{N}}\left(-u_{m}^{p}+u_{m}^{q_{m}(x)}\right) d x \leq \int_{\mathbb{R}^{N}} u_{0}^{q(x)} d x .
\end{aligned}
$$

Consequently we have $u_{0} \not \equiv 0$.

Proof of Proposition 9. We take a smooth radial function $\widehat{u}>$ 0 on $\mathbb{R}^{N}$. Since

$$
\begin{aligned}
J_{m}(K \widehat{u}) \leq & \frac{K^{p}}{p} \int_{\mathbb{R}^{N}}\left(|\nabla \widehat{u}|^{p}+|\widehat{u}|^{p}\right) d x \\
& -\int_{B_{R}} \frac{K^{q(x)}}{q(x)} \widehat{u}_{+}^{q(x)} d x \\
\leq & \frac{K^{p}}{p} \int_{\mathbb{R}^{N}}\left(|\nabla \widehat{u}|^{p}+|\widehat{u}|^{p}\right) d x \\
& -\frac{K^{p+C_{1}(\log R)^{\ell}}}{\operatorname{ess} \sup _{B_{R}} q(x)} \int_{B_{R}} \widehat{u}_{+}^{q(x)} d x \longrightarrow-\infty
\end{aligned}
$$

as $K \longrightarrow+\infty$, there exists $\widehat{K}>0$ independent of $m$ such that $J_{m}(\widehat{K} \widehat{u})<0$. If we set $\widehat{p}(t)=t \widehat{K} \widehat{u}$ for $t \in[0,1]$, then we see that

$$
\begin{aligned}
\widehat{p} & \in \widehat{P}=\left\{p \in C\left([0,1], W_{\text {rad }}^{1, p}\left(\mathbb{R}^{N}\right)\right) \mid p(0)\right. \\
& =0, p(1)=\widehat{K} \widehat{u}\} .
\end{aligned}
$$

Moreover, we have

$$
\begin{aligned}
\beta_{m} & =\inf _{p \in \widehat{P}} \max _{0 \leq t \leq 1} J_{m}(p(t)) \leq \max _{0 \leq t \leq 1} J_{m}(\widehat{p}(t)) \\
& =\max _{0 \leq t \leq \widehat{K}}\left[\frac{t^{p}}{p} \int_{\mathbb{R}^{N}}\left(|\nabla \widehat{u}|^{p}+|\widehat{u}|^{p}\right) d x\right. \\
& \left.-\int_{B_{R}} \frac{t^{q(x)}}{q(x)} \widehat{u}_{+}^{q(x)} d x\right] \leq C .
\end{aligned}
$$

On the other hand, since $u_{m}$ is a critical point of $J_{m}$ at $\beta_{m}$ we have

$$
\begin{aligned}
\beta_{m}= & \frac{1}{p} \int_{\mathbb{R}^{N}}\left(\left|\nabla u_{m}\right|^{p}+\left|u_{m}\right|^{p}\right) d x \\
& -\int_{\mathbb{R}^{N}} \frac{1}{q_{m}(x)}\left(u_{m}\right)_{+}^{q_{m}(x)} d x
\end{aligned}
$$

and, for any $\phi \in W_{\text {rad }}^{1, p}\left(\mathbb{R}^{N}\right)$,

$$
\begin{gathered}
\int_{\mathbb{R}^{N}}\left(\left|\nabla u_{m}\right|^{p-2} \nabla u_{m} \nabla \phi+\left|u_{m}\right|^{p-2} u_{m} \phi\right) d x \\
-\int_{\mathbb{R}^{N}}\left(u_{m}\right)_{+}^{q_{m}(x)-1} \phi d x=0 .
\end{gathered}
$$

In particular,

$$
\int_{\mathbb{R}^{N}}\left(\left|\nabla u_{m}\right|^{p}+\left|u_{m}\right|^{p}\right) d x-\int_{\mathbb{R}^{N}}\left(u_{m}\right)_{+}^{q_{m}(x)} d x=0 .
$$

From (54), (55), and (57), it follows that

$$
\int_{\mathbb{R}^{N}}\left(\frac{1}{p}-\frac{1}{q_{m}(x)}\right)\left(u_{m}\right)_{+}^{q_{m}(x)} d x \leq C .
$$

Furthermore, by $q(x) \leq q_{m}(x)$ we have

$$
\int_{\mathbb{R}^{N}}\left(\frac{1}{p}-\frac{1}{q(x)}\right)\left(u_{m}\right)_{+}^{q_{m}(x)} d x \leq C .
$$

Thus for any $L>0$ there exists a positive constant $C(L)$ such that

$$
\int_{B_{L}}\left(u_{m}\right)_{+}^{q_{m}(x)} d x \leq C(L) .
$$

Here, we take a constant $R_{0}>R$ sufficiently large (this $R_{0}$ will be chosen again later) and we have

$$
\left\|u_{m}\right\|_{W^{1, p}\left(\mathbb{R}^{N}\right)}^{p} \leq C\left(R_{0}\right)+\int_{\mathbb{R}^{N} \backslash B_{R_{0}}}\left(u_{m}\right)_{+}^{q_{m}(x)} d x
$$

by (57) and (60). Set $\delta=C_{1}\left(\log R_{0}\right)^{-\ell}$ and $A_{n, m}:=\left\{x \in B_{R_{0}^{n}} \backslash\right.$ $\left.B_{R_{0}^{n-1}} \mid q_{m}(x) \leq p+\delta\right\}$. Then we obtain

$$
\begin{aligned}
\int_{\mathbb{R}^{N} \backslash B_{R_{0}}}\left(u_{m}\right)_{+}^{q_{m}(x)} d x & \\
\leq & \int_{\left\{x \in \mathbb{R}^{N} \mid q_{m}(x)>p+\delta\right\}}\left(u_{m}\right)_{+}^{q_{m}(x)} d x \\
& +\int_{\left\{x \in \mathbb{R}^{N} \backslash B_{R_{0}} \mid q_{m}(x) \leq p+\delta\right\}}\left(u_{m}\right)_{+}^{q_{m}(x)} d x \\
\leq & \int_{\left\{x \in \mathbb{R}^{N} \mid q_{m}(x)>p+\delta\right\}}\left(u_{m}\right)_{+}^{q_{m}(x)} d x \\
& +\sum_{n=2}^{\infty} \int_{A_{n, m}}\left(u_{m}\right)_{+}^{p+C_{1}\left(n \log R_{0}\right)^{-\ell}} d x \\
& +\sum_{n=2}^{\infty} \int_{A_{n, m}}\left(u_{m}\right)_{+}^{p+\delta} d x=: L_{1}+L_{2}+L_{3},
\end{aligned}
$$

where the third inequality comes from the assumption (8). We shall estimate $L_{1}, L_{2}$, and $L_{3}$. For $L_{1}$, by (59) we have

$$
\begin{aligned}
& L_{1} \\
& \qquad \leq\left(\frac{1}{p}-\frac{1}{p+\delta}\right)^{-1} \int_{\mathbb{R}^{N}}\left(\frac{1}{p}-\frac{1}{q_{m}(x)}\right)\left(u_{m}\right)_{+}^{q_{m}(x)} d x \\
& \quad=C .
\end{aligned}
$$

In order to estimate $L_{2}$ and $L_{3}$, we prepare an estimate of $\left\|u_{m}\right\|_{L^{p}\left(A_{n, m}\right)}$. For each $n, m \in \mathbb{N}$ we have

$$
\int_{A_{n, m}} u_{m}^{p} d x \leq 2\left\|u_{m}\right\|_{L^{q_{m}(x)}\left(A_{n, m}\right)}^{p}\|1\|_{L^{r_{m}(x)}\left(A_{n, m}\right)}
$$


by the generalized Hölder inequality, where $r_{m}(x)$ := $q_{m}(x) /\left(q_{m}(x)-p\right)$. Now we assume $\left\|u_{m}\right\|_{L^{q_{m}(x)}\left(A_{n, m}\right)}>1$ and $\|1\|_{L^{r_{m}(x)}\left(A_{n, m}\right)}>1$ (if not, the proof is much simpler). By Proposition 2.2. in [27] we have

$$
\begin{aligned}
\left\|u_{m}\right\|_{L^{q_{m}(x)}\left(A_{n, m}\right)} & \leq\left(\int_{A_{n, m}} u_{m}^{q_{m}(x)} d x\right)^{\left(\operatorname{ess} \cdot \inf _{x \in A_{n, m}} q_{m}(x)\right)^{-1}} \\
& \leq\left(\int_{A_{n, m}} u_{m}^{q_{m}(x)} d x\right)^{1 /\left(p+\left(n \log R_{0}\right)^{-\ell}\right)}
\end{aligned}
$$

Since

$$
\begin{gathered}
\int_{A_{n, m}} u_{m}^{q_{m}(x)} d x \leq\left(\frac{1}{p}-\frac{1}{p+\left(n \log R_{0}\right)^{-\ell}}\right)^{-1} \\
\cdot \int_{\mathbb{R}^{N}}\left(\frac{1}{p}-\frac{1}{q_{m}(x)}\right) u_{m}^{q_{m}(x)} d x \leq C\left(n \log R_{0}\right)^{\ell},
\end{gathered}
$$

we obtain

$$
\left\|u_{m}\right\|_{L^{q_{m}(x)}\left(A_{n, m}\right)} \leq C\left(n \log R_{0}\right)^{\ell /\left(p+\left(n \log R_{0}\right)^{-\ell}\right)} .
$$

In the same way as above, we have

$$
\begin{aligned}
\|1\|_{L^{r_{m}(x)}\left(A_{n, m}\right)} & \leq\left(\int_{A_{n, m}} d x\right)^{\left(\operatorname{esssinf}_{x \in A_{n, m}} r_{m}(x)\right)^{-1}} \\
& \leq\left|A_{n, m}\right|^{1 /\left(1+p C_{1}^{-1}\left(\log R_{0}\right)^{\ell}\right)} \\
& \leq C R_{0}^{n /\left(1+p C_{1}^{-1}\left(\log R_{0}\right)^{\ell}\right)}
\end{aligned}
$$

where the second inequality comes from

$$
\begin{aligned}
\underset{x \in A_{n, m}}{\operatorname{essinf}} r_{m}(x) & =1+\frac{p}{\operatorname{esssup}_{x \in A_{n, m}} q_{m}(x)-p} \geq 1+\frac{p}{\delta} \\
& =1+p C_{1}^{-1}\left(\log R_{0}\right)^{\ell} .
\end{aligned}
$$

From (67) and (68) we obtain

$$
\begin{aligned}
& \int_{A_{n, m}} u_{m}^{p} d x \\
& \leq C R_{0}^{n /\left(1+p C_{1}^{-1}\left(\log R_{0}\right)^{\ell}\right)}\left(n \log R_{0}\right)^{p \ell /\left(p+\left(n \log R_{0}\right)^{-\ell}\right)} .
\end{aligned}
$$

For $L_{2}$, by using (70) and Proposition 6, we have

$$
\begin{aligned}
L_{2} & \leq C \sum_{n=2}^{\infty}\left\|u_{m}\right\|_{W^{1, p}\left(\mathbb{R}^{N}\right)}^{C_{1}\left(n \log R_{0}\right)^{-\ell}} R_{0}^{(-(N-1) / p)(n-1) C_{1}\left(n \log R_{0}\right)^{-\ell}} \int_{A_{n, m}} u_{m}^{p} d x \\
& \leq C\left\|u_{m}\right\|_{W^{1, p}\left(\mathbb{R}^{N}\right)}^{p} \sum_{n=2}^{\infty} R_{0}^{-\left((N-1) C_{1} / 2^{\ell} p\right)(n-1)^{1-\ell}\left(\log R_{0}\right)^{-\ell}}\left(\int_{A_{n, m}} u_{m}^{p} d x\right)^{C_{1}\left(n \log R_{0}\right)^{-\ell} / p} \\
& \leq C\left\|u_{m}\right\|_{W^{1, p}\left(\mathbb{R}^{N}\right)}^{p} \sum_{n=2}^{\infty} R_{0}^{-\left((N-1) C_{1} / 2^{\ell} p\right)(n-1)^{1-\ell}\left(\log R_{0}\right)^{-\ell}+C_{1} n^{1-\ell}\left(\log R_{0}\right)^{-\ell} /\left(p+p^{2} C_{1}^{-1}\left(\log R_{0}\right)^{-\ell}\right)}\left(n \log R_{0}\right)^{\left(\ell C_{1} /\left(p+\left(n \log R_{0}\right)^{-\ell}\right)\right)\left(n \log R_{0}\right)^{-\ell}} \\
& =C\left\|u_{m}\right\|_{W^{1, p}\left(\mathbb{R}^{N}\right)}^{p} \sum_{n=2}^{\infty} \delta_{1}\left(n, R_{0}\right)^{(n-1)^{1-\ell}} \delta_{2}\left(n, R_{0}\right) .
\end{aligned}
$$

Since

$$
\begin{array}{r}
\delta_{2}\left(n, R_{0}\right)=\left(n \log R_{0}\right)^{\left(\ell C_{1} /\left(p+\left(n \log R_{0}\right)^{-\ell}\right)\right)\left(n \log R_{0}\right)^{-\ell}} \longrightarrow 1 \\
\text { as } n \longrightarrow \infty \text { or } R_{0} \longrightarrow \infty,
\end{array}
$$

there exists a positive constant $\widetilde{C}$ which is independent of $n$ and $R_{0}$ such that

$$
\delta_{2}\left(n, R_{0}\right) \leq \widetilde{C} .
$$

On the other hand, for large $R_{0}$ we obtain

$$
\begin{aligned}
& \delta_{1}\left(n, R_{0}\right) \\
& =R_{0}^{-\left(C_{1} / p\right)\left(\log R_{0}\right)^{-\ell}\left[(N-1) / 2^{\ell}-\left(1 /\left(1+p C_{1}^{-1}\left(\log R_{0}\right)^{\ell}\right)\right)(n /(n-1))^{1-\ell}\right]} \\
& \leq R_{0}^{-\left(C_{1}(N-1) / 2^{\ell+1} p\right)\left(\log R_{0}\right)^{-\ell}}
\end{aligned}
$$

which yields

$$
\delta_{1}=\delta_{1}\left(n, R_{0}\right) \longrightarrow 0 \quad \text { uniformly in } n \text { as } R_{0} \longrightarrow \infty \text {. }
$$

From (73) and (75) we have

$$
\begin{array}{r}
L_{2} \leq C\left\|u_{m}\right\|_{W^{1, p}\left(\mathbb{R}^{N}\right)}^{p} \sum_{n=2}^{\infty} \delta_{1}^{(n-1)^{1-\ell}}=o(1)\left\|u_{m}\right\|_{W^{1, p}\left(\mathbb{R}^{N}\right)}^{p} \\
\text { as } R_{0} \longrightarrow \infty
\end{array}
$$

in the same way as the proof of Theorem 2. Thus for sufficiently large $R_{0}$ we have

$$
L_{2} \leq \frac{1}{3}\left\|u_{m}\right\|_{W^{1, p}\left(\mathbb{R}^{N}\right)}^{p} .
$$


In the same way as $L_{2}$, we obtain the estimate of $L_{3}$ for large $R_{0}$ as follows:

$$
\begin{aligned}
L_{3} \leq & C\left\|u_{m}\right\|_{W^{1, p}\left(\mathbb{R}^{N}\right)}^{\delta} \sum_{n=2}^{\infty} R_{0}^{(-(N-1) / p)(n-1) \delta} \int_{A_{n, m}} u_{m}^{p} d x \\
& \leq C\left\|u_{m}\right\|_{W^{1, p}\left(\mathbb{R}^{N}\right)}^{p} \\
& \cdot \sum_{n=2}^{\infty} R_{0}^{-((N-1) / p) C_{1}(n-1)\left(\log R_{0}\right)^{-\ell}}\left(\int_{A_{n, m}} u_{m}^{p} d x\right)^{\left(C_{1} / p\right)\left(\log R_{0}\right)^{-\ell}} \\
& \leq C\left\|u_{m}\right\|_{W^{1, p}\left(\mathbb{R}^{N}\right)}^{p} \\
& \cdot \sum_{n=2}^{\infty} R_{0}^{-((N-1) / p) C_{1}(n-1)\left(\log R_{0}\right)^{-\ell}+\left(n /\left(1+p C_{1}^{-1}\left(\log R_{0}\right)^{-\ell}\right)\right)\left(C_{1} / p\right)\left(\log R_{0}\right)^{-\ell}}(n \\
& \left.\cdot \log R_{0}\right)^{\ell C_{1} /\left(p\left(\log R_{0}\right)^{-\ell}+n^{-\ell}\right)} \leq C\left\|u_{m}\right\|_{W^{1, p}\left(\mathbb{R}^{N}\right)}^{p} \\
& \cdot \sum_{n=2}^{\infty} R_{0}^{-((N-1) / 2 p) C_{1}(n-1)\left(\log R_{0}\right)^{-\ell}}\left(n \log R_{0}\right)^{\ell C_{1} /\left(p\left(\log R_{0}\right)^{-\ell}+n^{-\ell}\right)} \\
& \leq C\left\|u_{m}\right\|_{W^{1, p}\left(\mathbb{R}^{N}\right)}^{p} \sum_{n=2}^{\infty} R_{0}^{-((N-1) / 4 p) C_{1}(n-1)\left(\log R_{0}\right)^{-\ell}},
\end{aligned}
$$

where the last inequality comes from

$$
\begin{aligned}
& \left(n \log R_{0}\right)^{\ell C_{1} /\left(p\left(\log R_{0}\right)^{\ell}+n^{-\ell}\right)} \\
& =o\left(R_{0}^{((N-1) / 4 p) C_{1}(n-1)\left(\log R_{0}\right)^{-\ell}}\right) \\
& \text { as } n \longrightarrow \infty \text { or } R_{0} \longrightarrow \infty .
\end{aligned}
$$

Therefore for sufficiently large $R_{0}$ we have

$$
L_{3} \leq \frac{1}{3}\left\|u_{m}\right\|_{W^{1, p}\left(\mathbb{R}^{N}\right)}^{p}
$$

From (61), (63), (77), and (80) we have

$$
\left\|u_{m}\right\|_{W^{1, p}\left(\mathbb{R}^{N}\right)}^{p} \leq C+\frac{2}{3}\left\|u_{m}\right\|_{W^{1, p}\left(\mathbb{R}^{N}\right)}^{p} .
$$

As a consequence $\left\{u_{m}\right\}$ is bounded.

\section{Mountain Pass Theorem under the Condition (C): Proof of Theorem 4}

In this section, we show Theorem 4 by a different method from Section 3.

Cerami [31] and Bartolo-Benci-Fortunato [32] have proposed a variant of (P.-S.) condition. In this paper, we use the condition (C) introduced by $[31,32]$ and the mountain pass theorem under the condition (C) (Theorem 11). Let $V$ be a real Banach space and $E \in C^{1}(V, \mathbb{R})$. First, we define the condition (C) based on $[31,32]$.

Definition 10 ([31, 32] Definition 1.1.). We say that $E$ satisfies the condition (C) in $\left(c_{1}, c_{2}\right),\left(-\infty \leq c_{1}<c_{2} \leq+\infty\right)$, if

(i) every bounded sequence $\left\{u_{k}\right\} \subset E^{-1}\left(\left(c_{1}, c_{2}\right)\right)$, for which $\left\{E\left(u_{k}\right)\right\}$ is bounded and $E^{\prime}\left(u_{k}\right) \longrightarrow 0$, possesses a convergent subsequence, and (ii) for any $c \in\left(c_{1}, c_{2}\right)$ there exist $\sigma, \rho, \alpha>0$ such that $[c-\sigma, c+\sigma] \subset\left(c_{1}, c_{2}\right)$ and for any $u \in E^{-1}([c-\sigma, c+\sigma])$ with $\|u\| \geq \rho,\left\|E^{\prime}(u)\right\|_{*}\|u\| \geq \alpha$.

Theorem 11 (mountain pass theorem under the condition (C)). Let E satisfy the condition $(C)$ in $(0,+\infty)$. Assume that

(i) $E(0)=0$

(ii) There exist $\rho>0, \alpha>0$ such that $E(u) \geq \alpha$ for any $u \in V$ with $\|u\|=\rho$.

(iii) There exists $u_{1} \in V$ such that $\left\|u_{1}\right\| \geq \rho$ and $E\left(u_{1}\right)<\alpha$. Define

$$
P=\left\{p \in C([0,1], V) \mid p(0)=0, p(1)=u_{1}\right\} .
$$

Then

$$
\beta=\inf _{p \in P} \sup _{0 \leq t \leq 1} E(p(t)) \geq \alpha
$$

is a critical value.

For $c \in \mathbb{R}$, we set

$$
\begin{aligned}
& E_{c}=\{u \in V \mid E(u)<c\}, \\
& K_{c}=\left\{u \in V \mid E^{\prime}(u)=0, E(u)=c\right\} .
\end{aligned}
$$

Note that Theorem 11 can be shown in the same way as the proof of Theorem 6.1 in p.109 in [35] by substituting the following deformation theorem under the condition $(\mathrm{C})$ for Theorem 3.4 in p.83 in [35].

Theorem 12 ([32] Theorem 1.3.). Let E satisfy the condition (C) in $\left(c_{1}, c_{2}\right)$. If $\beta \in\left(c_{1}, c_{2}\right)$ and $N$ is any neighborhood of $K_{\beta}$, there exist a bounded homeomorphism $\eta$ of $V$ onto $V$ and constants $\bar{\varepsilon}>\varepsilon>0$ such that $[\beta-\bar{\varepsilon}, \beta+\bar{\varepsilon}] \subset\left(c_{1}, c_{2}\right)$, satisfying the following properties:

(I) $\eta\left(E_{\beta+\varepsilon} \backslash N\right) \subset E_{\beta-\varepsilon}$

(II) $\eta\left(E_{\beta+\varepsilon}\right) \subset E_{\beta-\varepsilon}$ if $K_{\beta}=\emptyset$

(III) $\eta(u)=u$ if $|E(u)-\beta| \geq \bar{\varepsilon}$.

We set an energy functional from $W_{\text {rad }}^{1, p}\left(\mathbb{R}^{N}\right)$ to $\mathbb{R}$ as

$$
J(u)=\frac{1}{p} \int_{\mathbb{R}^{N}}\left(|\nabla u|^{p}+|u|^{p}\right) d x-\int_{\mathbb{R}^{N}} \frac{1}{q(x)} u_{+}^{q(x)} d x .
$$

We can check that $J \in C^{1}\left(W_{\text {rad }}^{1, p}\left(\mathbb{R}^{N}\right), \mathbb{R}\right)$ in the same way as the proof of Theorem 4 .

Proposition 13. Assume that $q(x)$ satisfies the hypotheses (7) and (8) in Theorem 2 and ess inf $x_{x \in B_{R}} q(x)>p$. Then $J$ satisfies the condition $(C)$ on $\mathbb{R}$.

Proof. We take $c_{1}, c_{2} \in \mathbb{R}$ with $c_{1}<c_{2}$ arbitrary. First, we shall show that $J$ satisfies (i) in Definition 10. Let $\left\{u_{m}\right\} \subset$ $W_{\text {rad }}^{1, p}\left(\mathbb{R}^{N}\right)$ be a bounded sequence satisfying $J\left(u_{m}\right) \in\left(c_{1}, c_{2}\right)$ 
and $\left\|J^{\prime}\left(u_{m}\right)\right\|_{*} \longrightarrow 0$ as $m \longrightarrow+\infty$. Then the following holds true for any $\phi \in W_{\text {rad }}^{1, p}\left(\mathbb{R}^{N}\right)$ :

$$
\begin{gathered}
\int_{\mathbb{R}^{N}}\left(\left|\nabla u_{m}\right|^{p-2} \nabla u_{m} \nabla \phi+\left|u_{m}\right|^{p-2} u_{m} \phi\right) d x \\
-\int_{\mathbb{R}^{N}}\left(u_{m}\right)_{+}^{q(x)-1} \phi d x=o(1) .
\end{gathered}
$$

In particular, since $\left\{u_{m}\right\}$ is bounded it follows that

$$
\int_{\mathbb{R}^{N}}\left(\left|\nabla u_{m}\right|^{p}+\left|u_{m}\right|^{p}\right) d x-\int_{\mathbb{R}^{N}}\left(u_{m}\right)_{+}^{q(x)} d x=o(1) .
$$

Likewise since $\left\{u_{m}\right\}$ is bounded, there exists a subsequence written as $\left\{u_{m}\right\}$ for simplicity and $u_{0} \in W_{\text {rad }}^{1, p}\left(\mathbb{R}^{N}\right)$ such that $u_{m} \rightarrow u_{0}$ weakly in $W^{1, p}\left(\mathbb{R}^{N}\right)$. Put

$$
\begin{aligned}
G_{m} & \\
= & \left\langle\left|\nabla u_{m}\right|^{p-2} \nabla u_{m}-\left|\nabla u_{0}\right|^{p-2} \nabla u_{0}, \nabla u_{m}-\nabla u_{0}\right\rangle_{\mathbb{R}^{N}} \\
& +\left(u_{m}^{p-1}-u_{0}^{p-1}\right)\left(u_{m}-u_{0}\right)
\end{aligned}
$$

as in Section 3. In the same way as Step 2 in the proof of Theorem 4 in Section 3 by substituting (86) and (87) for (56) and (57), respectively, we have

$$
\int_{\mathbb{R}^{N}} G_{m} d x=o(1)
$$

as $m \longrightarrow \infty$ by Theorem 2 . Recalling that

$$
\begin{aligned}
& \left\langle|b|^{p-2} b-|a|^{p-2} a, b-a\right\rangle \\
& \geq \begin{cases}2^{2-p}|b-a|^{p} & \text { if } p \geq 2, \\
(p-1)|b-a|^{2}\left(1+|a|^{2}+|b|^{2}\right)^{(p-2) / 2} & \text { if } 1 \leq p \leq 2,\end{cases}
\end{aligned}
$$

consequently we have

$$
\begin{aligned}
& \lim _{m \rightarrow \infty} \int_{\mathbb{R}^{N}}\left(\left|\nabla\left(u_{m}-u_{0}\right)\right|^{p}+\left|u_{m}-u_{0}\right|^{p}\right) d x \\
& \leq C_{m \longrightarrow \infty} \lim _{\mathbb{R}^{N}} G_{m} d x=0 .
\end{aligned}
$$

This implies that $u_{m} \longrightarrow u_{0}$ strongly in $W^{1, p}\left(\mathbb{R}^{N}\right)$.

Next, we shall show (ii). For any $c \in\left(c_{1}, c_{2}\right)$, we take some $\sigma$ with $[c-\sigma, c+\sigma] \subset\left(c_{1}, c_{2}\right)$. We will choose suitable $\rho>0$ again later. By deriving a contradiction, we show that there exists $\alpha>0$ such that for any $u \in J^{-1}([c-\sigma, c+\sigma])$ with $\|u\| \geq \rho,\left\|J^{\prime}(u)\right\|_{*}\|u\| \geq \alpha$. We assume that there exists $\left\{u_{m}\right\} \subset W_{\text {rad }}^{1, p}\left(\mathbb{R}^{N}\right)$ such that $u_{m} \in J^{-1}([c-\sigma, c+\sigma])$ with $\left\|u_{m}\right\|_{W^{1, p\left(\mathbb{R}^{N}\right)}} \geq \rho$ and $\left\|J^{\prime}\left(u_{m}\right)\right\|_{*}\left\|u_{m}\right\|_{W^{1, p\left(\mathbb{R}^{N}\right)}}=: \alpha_{m} \longrightarrow 0$ as $m \longrightarrow+\infty$. Since $J^{\prime}\left(u_{m}\right) u_{m} \longrightarrow 0$ as $m \longrightarrow+\infty$, we have

$$
\left|\left\|u_{m}\right\|_{W^{1, p}\left(\mathbb{R}^{N}\right)}^{p}-\int_{\mathbb{R}^{N}}\left(u_{m}\right)_{+}^{q(x)} d x\right| \leq \alpha_{m}
$$

which yields

$$
c+\sigma \geq J\left(u_{m}\right) \geq \int_{\mathbb{R}^{N}}\left(\frac{1}{p}-\frac{1}{q(x)}\right)\left(u_{m}\right)_{+}^{q(x)} d x-\alpha_{m} .
$$

Moreover, in the same way as the proof of Proposition 9, for large $m$ we have

$$
\begin{aligned}
& \int_{A_{n}} u_{m}^{p} d x \\
& \quad \leq C R_{0}^{n /\left(1+p C_{1}^{-1}\left(\log R_{0}\right)^{\ell}\right)}\left(n \log R_{0}\right)^{p \ell /\left(p+\left(n \log R_{0}\right)^{-\ell}\right)},
\end{aligned}
$$

where $A_{n}:=\left\{x \in B_{R_{0}^{n}} \backslash B_{R_{0}^{n-1}} \mid q(x) \leq p+\delta\right\}$ for $n \geq 2$ and $R_{0}$ is the same as the proof of Proposition 9. By substituting (93) and (94) for (59) and (70), we obtain the following estimates:

$$
\begin{aligned}
\left\|u_{m}\right\|_{W^{1, p}\left(\mathbb{R}^{N}\right)}^{p}-\alpha_{m} \leq & \int_{B_{R_{0}}}\left(u_{m}\right)_{+}^{q(x)} d x \\
& +\int_{\mathbb{R}^{N} \backslash B_{R_{0}}}\left(u_{m}\right)_{+}^{q(x)} d x \\
\leq & C\left(R_{0}\right)\left(c+\sigma+\alpha_{m}\right) \\
& +\frac{2}{3}\left\|u_{m}\right\|_{W^{1, p}\left(\mathbb{R}^{N}\right)}^{p},
\end{aligned}
$$

where $C\left(R_{0}\right)$ is a positive constant independent of $\rho$. Therefore we have

$$
\begin{aligned}
\left\|u_{m}\right\|_{W^{1, p}\left(\mathbb{R}^{N}\right)}^{p} & \leq 3\left\{\alpha_{m}+C\left(R_{0}\right)\left(c+\sigma+\alpha_{m}\right)\right\} \\
& \leq 3\left\{1+C\left(R_{0}\right)\left(c_{2}+1\right)\right\}
\end{aligned}
$$

for large $m$. If we choose sufficiently large $\rho$ satisfying $\rho>$ $3^{1 / p}\left\{1+C\left(R_{0}\right)\left(c_{2}+1\right)\right\}^{1 / p}$, then we see that (96) contradicts $\left\|u_{m}\right\|_{W^{1, p}\left(\mathbb{R}^{N}\right)} \geq \rho$.

The proof of Proposition 13 is now complete.

Proposition 14. Assume that $q(x)$ satisfies the hypotheses (7) and (8) in Theorem 2 and ess $\inf _{x \in B_{R}} q(x)>p$. Then $J$ has the mountain pass geometry, that is, $J$ satisfies (i), (ii), and (iii) in Theorem 11.

Proof. (i) is obvious. We prove (ii). Let $S$ be the best constant of the Sobolev inequality: $S\|v\|_{L^{p^{*}}\left(\mathbb{R}^{N}\right)}^{p} \leq\|\nabla v\|_{L^{p}\left(\mathbb{R}^{N}\right)}^{p}$ for $v \in$ $C_{c}^{\infty}\left(\mathbb{R}^{N}\right)$. Set $q^{*}=\max \left\{p^{*}, p^{2},\|q\|_{L^{\infty}\left(\mathbb{R}^{N}\right)}\right\}$. Note that $q^{*} \geq$ $p^{*}>p N /(N-1)$. For $u \in W_{\text {rad }}^{1, p}\left(\mathbb{R}^{N}\right)$ with $\|u\|_{W^{1, p}\left(\mathbb{R}^{N}\right)}=\gamma$, it follows that

$$
\begin{aligned}
& \int_{\mathbb{R}^{N}} \frac{1}{q(x)} u_{+}^{q(x)} d x \leq \frac{1}{p} \int_{\mathbb{R}^{N}}|u|^{p} d x+\frac{1}{p}\left[\int_{B_{r}}|u|^{p^{*}} d x\right. \\
& \left.+\int_{\mathbb{R}^{N} \backslash B_{r}}|u|^{q^{*}} d x\right] \leq \frac{1}{p} \int_{\mathbb{R}^{N}}|u|^{p} d x \\
& +\frac{1}{p}\left[\left(S^{-1} \int_{\mathbb{R}^{N}}|\nabla u|^{p} d x\right)^{p^{*} / p}\right. \\
& \left.+\|u\|_{L^{p}\left(\mathbb{R}^{N}\right)}^{q^{*}((p-1) / p)}\|\nabla u\|_{L^{p}\left(\mathbb{R}^{N}\right)}^{q^{*} / p} K(r)\right] \leq \frac{1}{p} \int_{\mathbb{R}^{N}}|u|^{p} d x \\
& +\frac{1}{p} \int_{\mathbb{R}^{N}}|\nabla u|^{p} d x\left[S^{-p^{*} / p} \gamma^{p^{*}-p}+K(r) \gamma^{q^{*}-p}\right],
\end{aligned}
$$


where $K(r)=\left(p / \omega_{N-1}\right)^{q^{*} / p} \int_{\mathbb{R}^{N} \backslash B_{r}}|x|^{-q^{*}(N-1) / p} d x<\infty$ and the second inequality comes from Proposition 6. From this if $\gamma$ is sufficiently small, we have

$$
\begin{aligned}
& J(u) \\
& \quad \geq \frac{1}{p} \int_{\mathbb{R}^{N}}|\nabla u|^{p} d x\left[1-S^{-p^{*} / p} \gamma^{p^{*}-p}-K(r) \gamma^{q^{*}-p}\right] \\
& \quad>0 .
\end{aligned}
$$

For $\left\{u_{m}\right\} \subset W_{\text {rad }}^{1, p}\left(\mathbb{R}^{N}\right)$ and $\gamma$ satisfying $\left\|u_{m}\right\|_{W^{1, p}\left(\mathbb{R}^{N}\right)}=\gamma$ and (98), we assume that $J\left(u_{m}\right) \longrightarrow 0$ and derive a contradiction. From (98) it follows that $\int_{\mathbb{R}^{N}}\left|\nabla u_{m}\right|^{p} d x \longrightarrow 0$. In addition, for sufficiently large $R$ we have

$$
\begin{aligned}
& \int_{\mathbb{R}^{N}} \frac{1}{q(x)}\left(u_{m}\right)_{+}^{q(x)} d x \leq \frac{1}{p}\left(\int_{B_{r}}\left|u_{m}\right|^{q(x)} d x\right. \\
& \left.\quad+\int_{B_{R} \backslash B_{r}}\left|u_{m}\right|^{q(x)} d x+\int_{\mathbb{R}^{N} \backslash B_{R}}\left|u_{m}\right|^{q(x)} d x\right) \\
& \quad \leq \frac{1}{p}\left[\int_{B_{r}}\left|u_{m}\right|^{p^{*}} d x+\int_{B_{R}}\left|u_{m}\right|^{p} d x\right. \\
& \left.\quad+\int_{\mathbb{R}^{N} \backslash B_{r}}\left|u_{m}\right|^{q^{*}} d x+\int_{\mathbb{R}^{N} \backslash B_{R}}\left|u_{m}\right|^{p+C_{1}(\log |x|)^{-\ell}} d x\right] \\
& \quad=\frac{1}{p}\left(H_{1}+H_{2}+H_{3}+H_{4}\right) .
\end{aligned}
$$

By using the estimates in the calculation of $\int_{\mathbb{R}^{N}}(u)_{+}^{q(x)} / q(x) d x$ to show (98) we have $H_{1}=o(1)$ and $H_{3}=o(1)$ as $m \longrightarrow \infty$. For $\mathrm{H}_{2}$ we have

$$
H_{2} \leq\left|B_{R}\right|^{1-p / p^{*}} S^{-1} \int_{\mathbb{R}^{N}}\left|\nabla u_{m}\right|^{p} d x=o(1) .
$$

We can show that $H_{4}$ is bounded uniformly for $m$ and $H_{4} \longrightarrow$ 0 as $R \longrightarrow \infty$ in the same way as the estimate of $I_{3}(j, K)$ in the proof of Theorem 2. Therefore

$$
\int_{\mathbb{R}^{N}} \frac{1}{q(x)}\left|u_{m}\right|^{q(x)} d x \rightarrow 0
$$

as $m \longrightarrow \infty$, which implies that $\left\|u_{m}\right\|_{W^{1, p}\left(\mathbb{R}^{N}\right)} \longrightarrow 0$ since $J\left(u_{m}\right) \longrightarrow 0$ as $m \longrightarrow \infty$. This contradicts $\left\|u_{m}\right\|_{W^{1, p}\left(\mathbb{R}^{N}\right)}=\gamma$.

Finally, we prove (iii). We take a smooth radial function $v$ such that $\|v\|_{W^{1, p}\left(\mathbb{R}^{N}\right)}=\gamma, v>0$ in $B_{R}$, where $R$ is in the hypothesis (8). Recall that $q:=\operatorname{ess~inf}_{x \in B_{R}} q(x)>p$. By taking sufficiently large $t$ we have

$$
\begin{aligned}
J(t v)= & \frac{t^{p}}{p} \int_{\mathbb{R}^{N}}\left(|\nabla v|^{p}+|v|^{p}\right) d x-\int_{\mathbb{R}^{N}} \frac{t^{q(x)}}{q(x)} v_{+}^{q(x)} d x \\
\leq & \frac{t^{p}}{p} \int_{\mathbb{R}^{N}}\left(|\nabla v|^{p}+|v|^{p}\right) d x \\
& -t^{q} \int_{B_{R}} \frac{1}{q(x)} v_{+}^{q(x)} d x<0 .
\end{aligned}
$$

Since $\|t v\|_{W^{1, p\left(\mathbb{R}^{N}\right)}}>\gamma$ we prove (iii).
Proof of Theorem 4. From Propositions 13 and 14 and Theorem 11, we can show the existence of a nontrivial critical point $u \in W_{\text {rad }}^{1, p}\left(\mathbb{R}^{N}\right)$ which is a weak solution to $-\Delta_{p} u+$ $|u|^{p-2} u=u_{+}^{q(x)-1}$ in $\mathbb{R}^{N}$. Then we also see that $u \geq 0$ in $\mathbb{R}^{N}$.

\section{Appendix}

In this section we show Propositions 6 and 7.

Proof of Proposition 6. It is sufficient to show that (13) holds for $f \in C_{c}^{\infty}\left(\mathbb{R}^{N}\right)$ with radially symmetric. We have

$$
r^{N-1}|f(r)|^{p}=-\int_{r}^{\infty} \frac{d}{d s}\left(s^{N-1}|f(s)|^{p}\right) d s .
$$

By direct calculation we have

$$
\begin{aligned}
\left(s^{N-1}|f(s)|^{p}\right)^{\prime}= & (N-1) s^{N-2}|f(s)|^{p} \\
& +p s^{N-1}|f(s)|^{p-2} f(s) f(s)^{\prime} .
\end{aligned}
$$

Thus it follows that

$$
\begin{aligned}
r^{N-1} & |f(r)|^{p} \\
= & -(N-1) \int_{r}^{\infty} s^{N-2}|f(s)|^{p} d s \\
& \quad-p \int_{r}^{\infty} s^{N-1}|f(s)|^{p-2} f(s) f(s)^{\prime} d s \\
\leq & p \int_{r}^{\infty} s^{N-1}|f(s)|^{p-1}\left|f(s)^{\prime}\right| d s \\
\leq & \frac{p}{\omega_{N-1}}\|f\|_{L^{p}\left(\mathbb{R}^{N}\right)}^{p-1}\|\nabla f\|_{L^{p}\left(\mathbb{R}^{N}\right)} .
\end{aligned}
$$

Consequently (13) follows immediately.

Proof of Proposition 7. By (13) we have

$$
\begin{aligned}
\int_{\mathbb{R}^{N} \backslash B_{R}}|u|^{q} d x & \leq C_{u} \int_{\mathbb{R}^{N} \backslash B_{R}}|x|^{-((N-1) / p) q} d x \\
& =C_{u} \int_{R}^{\infty} r^{-(N-1)(q / p-1)} d r,
\end{aligned}
$$

where $C_{u}=\left(p / \omega_{N-1}\right)^{q / p}\|u\|_{L^{p}\left(\mathbb{R}^{N}\right)}^{q(p-1) / p}\|\nabla u\|_{L^{p}\left(\mathbb{R}^{N}\right)}^{q / p}$. When $(N-$ $1)(q / p-1)>1$, that is, $q>p N /(N-1)$, we have

$$
\int_{\mathbb{R}^{N} \backslash B_{R}}|u|^{q} d x \leq C_{u} R^{-(N-1)(q / p-1)+1} .
$$

Let $\left\{u_{m}\right\}$ be a sequence such that $u_{m} \rightarrow 0$ weakly in $W_{\text {rad }}^{1, p}\left(\mathbb{R}^{N}\right)$. Firstly we show that the case of $q \in(p N /(N-$ $\left.1), p^{*}\right)$. In this case we have

$$
\int_{\mathbb{R}^{N}}\left|u_{m}\right|^{q} d x \leq \int_{B_{R}}\left|u_{m}\right|^{q} d x+C_{u_{m}} R^{-(N-1)(q / p-1)+1} .
$$


Since $C_{u_{m}}$ is bounded from above uniformly, letting $m \longrightarrow \infty$ and $R \longrightarrow \infty$ we have $u_{m} \longrightarrow 0$ strongly in $L^{q}\left(\mathbb{R}^{N}\right)$.

Next, for $q \in(p, p N /(N-1)]$ using interpolation of $L^{q}$ space, we have

$$
\left\|u_{m}\right\|_{L^{q}\left(\mathbb{R}^{N}\right)} \leq\left\|u_{m}\right\|_{L^{p}\left(\mathbb{R}^{N}\right)}^{\lambda}\left\|u_{m}\right\|_{L^{r}\left(\mathbb{R}^{N}\right)}^{1-\lambda},
$$

where $r \in\left(p N /(N-1), p^{*}\right)$. Since $\left\|u_{m}\right\|_{L^{r}\left(\mathbb{R}^{N}\right)} \longrightarrow 0$ and $\left\|u_{m}\right\|_{L^{p}\left(\mathbb{R}^{N}\right)}$ is bounded we have $\left\|u_{m}\right\|_{L^{q}\left(\mathbb{R}^{N}\right)} \longrightarrow 0$.

\section{Data Availability}

The data used to support the findings of this study are available from the corresponding author upon request.

\section{Disclosure}

An earlier abstract of this manuscript was presented in Osaka University Differential Equation Seminar in 2017.

\section{Conflicts of Interest}

The authors declare that they have no conflicts of interest.

\section{Acknowledgments}

The authors are grateful to Professor Michinori Ishiwata (Osaka University) for helpful advice on this subject. Part of this work was supported by JSPS Grant-in-Aid for Fellows (DC2), no. 16J08945 (Masato Hashizume) and no. 16J07472 (Megumi Sano).

\section{References}

[1] C. O. Alves and J. L. Barreiro, "Multiple solutions for a class of quasilinear problems involving variable exponents," Asymptotic Analysis, vol. 96, no. 2, pp. 161-184, 2016.

[2] Y. Fu and X. Zhang, "A multiplicity result for $p(x)$-Laplacian problem in $\mathbb{R}^{N}$," Nonlinear Analysis, vol. 70 , no. 6, pp. 22612269, 2009.

[3] C. O. Alves and M. C. Ferreira, "Existence of solutions for a class of $p(x)$-Laplacian equations involving a concave-convex nonlinearity with critical growth in $\mathbb{R}^{N}$," Topological Methods in Nonlinear Analysis, vol. 45, no. 2, pp. 399-422, 2015.

[4] X. L. Fan and X. Han, "Existence and multiplicity of solutions for $p(x)$-Laplacian equations in $\mathbb{R}^{N}$," Nonlinear Analysis. Theory, Methods \& Applications, vol. 59, no. 1-2, pp. 173-188, 2004.

[5] X. Fan, " $p(x)$-Laplacian equations in $\mathbb{R}^{N}$ with periodic data and nonperiodic perturbations," Journal of Mathematical Analysis and Applications, vol. 341, no. 1, pp. 103-119, 2008.

[6] X. Zhang, "A minimization problem with variable growth on Nehari manifold," Monatshefte für Mathematik, vol. 181, no. 2, pp. 485-500, 2016.

[7] C. O. Alves and M. A. Souto, "Existence of solutions for a class of problems in $\mathbb{R}^{N}$ involving the $p(x)$-Laplacian," in Contributions to nonlinear analysis, vol. 66 of Progress in Nonlinear Differential Equations and Their Applications, pp. 17-32, Birkhäuser, Basel, Switzerland, 2006.
[8] N. Saintier and A. Silva, "Local existence conditions for an equations involving the $p(x)$-Laplacian with critical exponent in $\mathbb{R}^{N}$," Nonlinear Differential Equations and Applications NoDEA, vol. 24, no. 2, Art. 19, 36 pages, 2017.

[9] O. Kovácik and J. Rákosnk, "On spaces $L^{p}(x)$ and $W^{k, p}(x)$," Czechoslovak Mathematical Journal, vol. 41, no. 116, pp. 592-618, 1991.

[10] B. Cekic, R. Mashiyev, and G. T. Alisoy, "On the Sobolevtype inequality for Lebesgue spaces with a variable exponent," International Mathematical Forum. Journal for Theory and Applications, vol. 1, no. 25-28, pp. 1313-1323, 2006.

[11] R. Mashiyev and B. Cekic, "Sobolev-type inequality for spaces $L^{p}(x)\left(\mathbb{R}^{N}\right)$," International Journal of Contemporary Mathematical Sciences, vol. 2, no. 9-12, pp. 423-429, 2007.

[12] D. E. Edmunds and J. Rakosnik, "Sobolev embeddings with variable exponent," Studia Mathematica, vol. 143, no. 3, pp. 267293, 2000.

[13] D. E. Edmunds and J. Rakosnik, "Sobolev embeddings with variable exponent, II," Mathematische Nachrichten, vol. 246/247, pp. 53-67, 2002.

[14] L. Diening, "Riesz potential and SOBolev embeddings on generalized Lebesgue and SOBolev spaces $L^{p(\cdot)}$ and $W^{k, p(\cdot)}$," Mathematische Nachrichten, vol. 268, pp. 31-43, 2004.

[15] C. Capone, D. Cruz-Uribe, and A. Fiorenza, "The fractional maximal operator and fractional integrals on variable $L^{p}$ spaces," Revista Matemática Iberoamericana, vol. 23, no. 3, pp. 743-770, 2007.

[16] P. Harjulehto and P. Hästö, "Sobolev inequalities with variable exponent attaining the values 1 and $n$," Publicacions Matemátiques, vol. 52, no. 2, pp. 347-363, 2008.

[17] K. Kurata and N. Shioji, "Compact embedding from $W_{0}^{1,2}(\Omega)$ to $L^{q(x)}(\Omega)$ and its application to nonlinear elliptic boundary value problem with variable critical exponent," Journal of Mathematical Analysis and Applications, vol. 339, no. 2, pp. 1386-1394, 2008.

[18] X. L. Fan, J. S. Shen, and D. Zhao, "Sobolev embedding theorems for spaces $W^{k, p(x)}(\Omega)$," Journal of Mathematical Analysis and Applications, vol. 262, no. 2, pp. 749-760, 2001.

[19] Y. Mizuta, T. Ohno, T. Shimomura, and N. Shioji, "Compact embeddings for Sobolev spaces of variable exponents and existence of solutions for nonlinear elliptic problems involving the $p(x)$-Laplacian and its critical exponent," Annales Academice Scientiarum Fennica, vol. 35, no. 1, pp. 115-130, 2010.

[20] D. V. Cruz-Uribe and A. Fiorenza, Variable Lebesgue Spaces: Foundations and Harmonic Analysis, Applied and Numerical Harmonic Analysis, Birkhäuser, Basel, Switzerland, 2013.

[21] J. Fernández Bonder, N. Saintier, and A. Silva, "On the Sobolev embedding theorem for variable exponent spaces in the critical range," Journal of Differential Equations, vol. 253, no. 5, pp. 1604-1620, 2012.

[22] J. Fernández Bonder, N. Saintier, and A. Silva, "On the Sobolev trace theorem for variable exponent spaces in the critical range," Annali di Matematica Pura ed Applicata. Series IV, vol. 193, no. 6, pp. 1607-1628, 2014.

[23] W. A. Strauss, "Existence of solitary waves in higher dimensions," Communications in Mathematical Physics, vol. 55, no. 2, pp. 149-162, 1977.

[24] P.-L. Lions, "Symétrie et compacité dans les espaces de Sobolev," Journal of Functional Analysis, vol. 49, no. 3, pp. 315-334, 1982.

[25] J. Chabrowski, "On compact embeddings of radial Sobolev spaces and their applications," Communications in Partial Differential Equations, vol. 17, no. 5-6, pp. 941-952, 1992. 
[26] Y. Ebihara and T. P. Schonbek, "On the (non)compactness of the radial Sobolev spaces," Hiroshima Mathematical Journal, vol. 16, no. 3, pp. 665-669, 1986.

[27] X. Fan, Y. Zhao, and D. Zhao, "Compact imbedding theorems with symmetry of Strauss-Lions type for the space $W^{k, p(x)}(\Omega)$," Journal of Mathematical Analysis and Applications, vol. 255, no. 1, pp. 333-348, 2001.

[28] E. DiBenedetto, " $C^{1+\alpha}$ local regularity of weak solutions of degenerate elliptic equations," Nonlinear Analysis, vol. 7, no. 8, pp. 827-850, 1983.

[29] P. Pucci and J. Serrin, The Maximum Principle, vol. 73 of Progress in Nonlinear Differential Equations and their Applications, Birkhauser Verlag, Basel, Switzerland, 2007.

[30] Z. Liu and Z.-Q. Wang, "On the Ambrosetti-Rabinowitz superlinear condition," Advanced Nonlinear Studies, vol. 4, no. 4, pp. 563-574, 2004.

[31] G. Cerami, "An existence criterion for the critical points on unbounded manifolds," Istituto Lombardo. Accademia di Scienze e Lettere. Rendiconti. Scienze Matematiche, Fisiche, Chimiche e Geologiche. A, vol. 112, no. 2, pp. 332-336 (1979), 1978.

[32] P. Bartolo, V. Benci, and D. Fortunato, "Abstract critical point theorems and applications to some nonlinear problems with 'strong' resonance at infinity,' Nonlinear Analysis. Theory, Methods \& Applications, vol. 7, no. 9, pp. 981-1012, 1983.

[33] H. Brezis, Functional Analysis, Sobolev Spaces and Partial Differential Equations, Universitext, Springer, New York, NY, USA, 2011.

[34] P. H. Rabinowitz, Minimax Methods in Critical Point Theory with Applications to Differential Equations, vol. 65 of CBMS Regional Conference Series in Mathematics, American Mathematical Society, Providence, RI, USA, 1986.

[35] M. Struwe, Variational Methods. Applications to Nonlinear Partial Differential Equations and Hamiltonian Systems, vol. 34 of Modern Surveys in Mathematics, Springer, Berlin, Germany, 4th edition, 2008.

[36] A. Ambrosetti and P. H. Rabinowitz, "Dual variational methods in critical point theory and applications," Journal of Functional Analysis, vol. 14, pp. 349-381, 1973. 


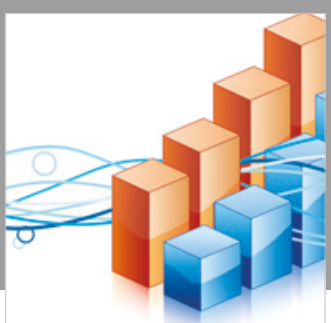

Advances in

Operations Research

\section{-n-m}
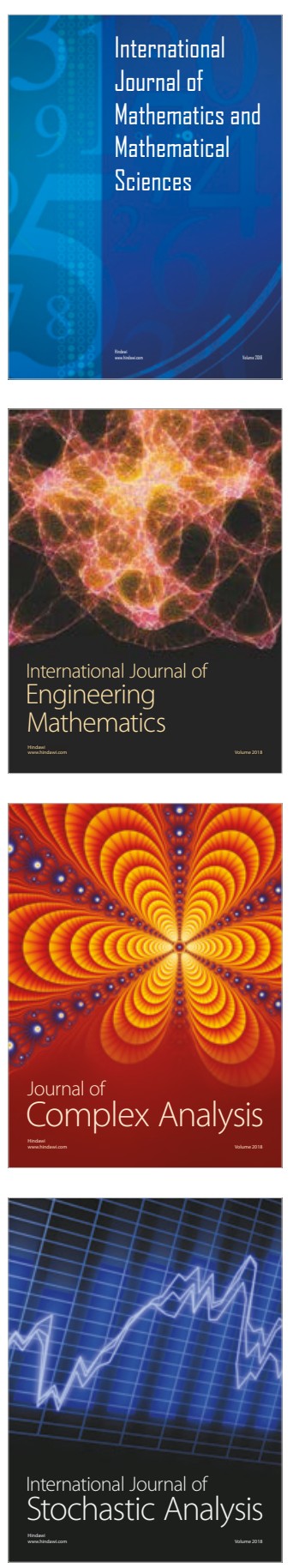
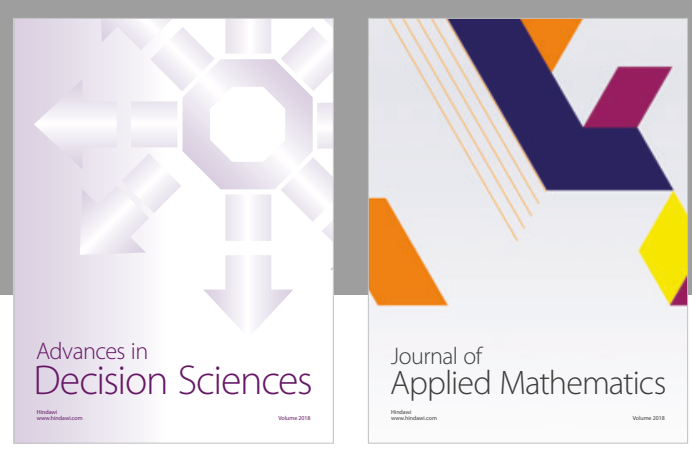

Journal of

Applied Mathematics
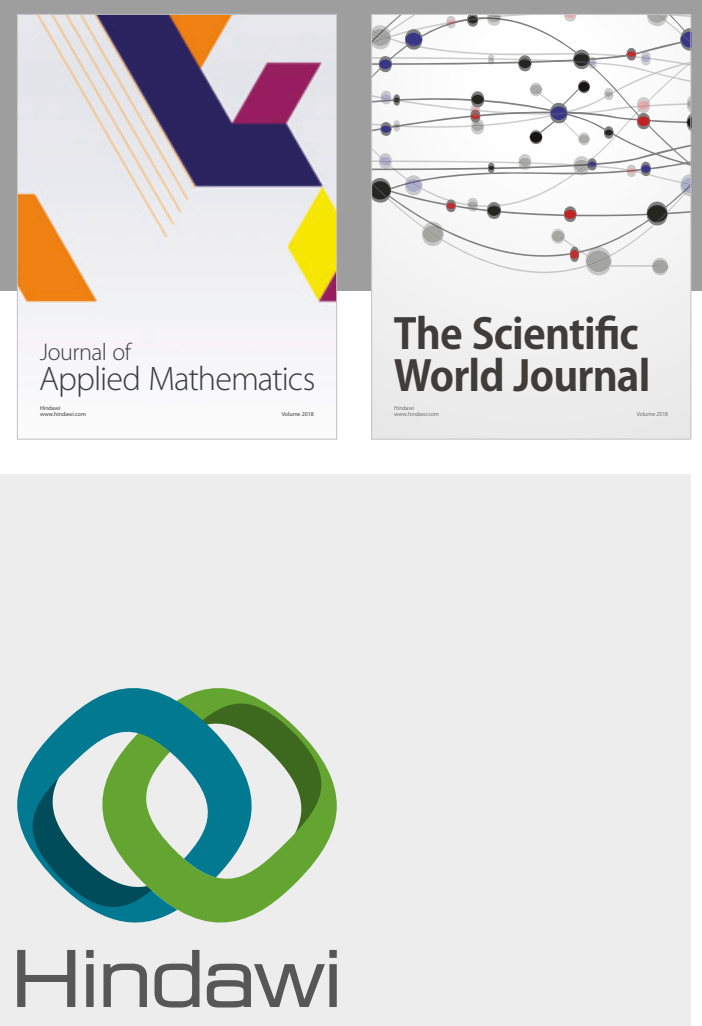

Submit your manuscripts at

www.hindawi.com

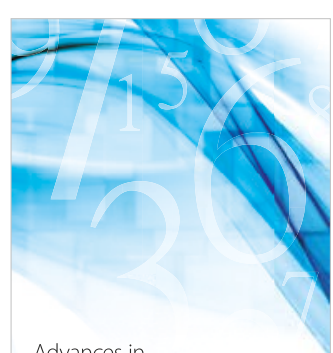

Advances in
Numerical Analysis
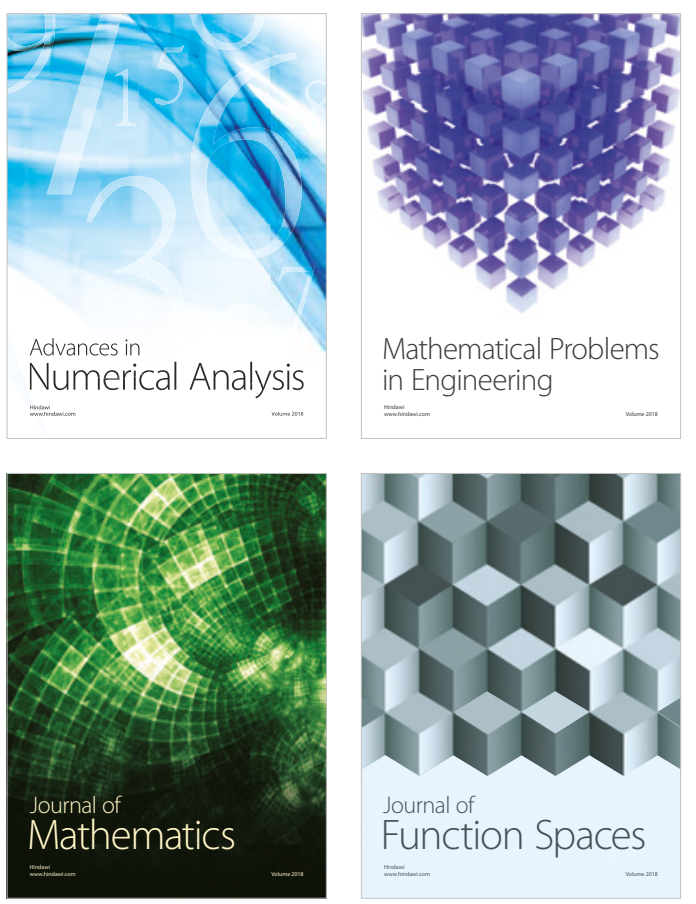

Mathematical Problems in Engineering

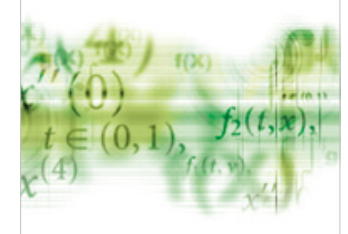

International Journal of

Differential Equations

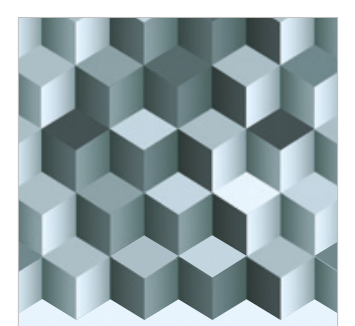

Journal of

Function Spaces

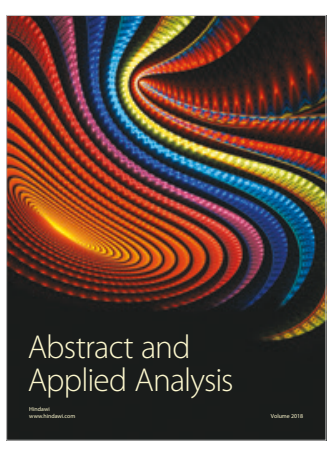

The Scientific

World Journal

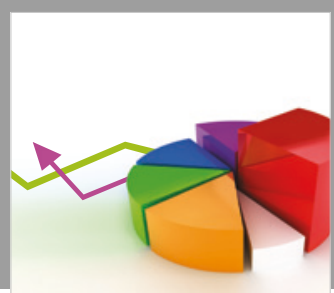

Journal of

Probability and Statistics
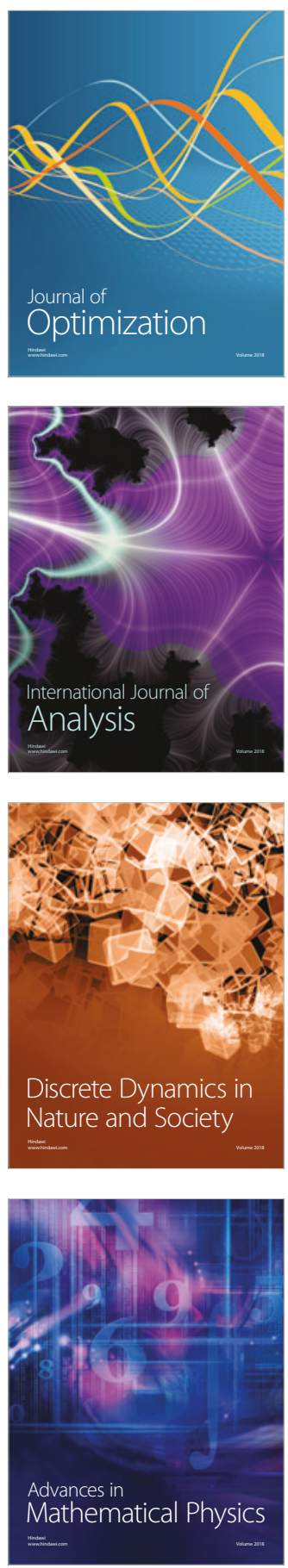